\title{
Coupling of gravity to matter, spectral action and cosmic topology
}

\author{
Branimir Ćaćić, Matilde Marcolli, and Kevin Teh
}

\begin{abstract}
We consider a model of modified gravity based on the spectral action functional, for a cosmic topology given by a spherical space form, and the associated slow-roll inflation scenario. We consider then the coupling of gravity to matter determined by an almost-commutative geometry over the spherical space form. We show that this produces a multiplicative shift of the amplitude of the power spectra for the density fluctuations and the gravitational waves, by a multiplicative factor equal to the total number of fermions in the matter sector of the model. We obtain the result by an explicit nonperturbative computation, based on the Poisson summation formula and the spectra of twisted Dirac operators on spherical space forms, as well as, for more general spacetime manifolds, using a heat kernel computation.
\end{abstract}

Mathematics Subject Classification (2010). 83F05, 83C65, 58J42.

Keywords. Spectral action, cosmic topology, gravity coupled to matter, Poisson summation formula, heat kernel.

\section{Introduction}

Models of gravity coupled to matter based on Noncommutative Geometry are usually obtained (see [35], [5], [8], [11]) by considering an underlying geometry given by a product $X \times F$ of an ordinary 4-dimensional (Riemannian compact) spacetime manifold and a finite noncommutative space $F$.

The main purpose of the paper is to show how the slow-roll inflation potential derived in [20], [21] is affected by the presence of the matter content and the almostcommutative geometry. We first consider the case of spherical space forms using the Poisson summation formula technique and the nonperturbative calculation of the spectral action, and then the case of more general spacetime manifolds using a nonperturbative heat kernel argument, to show that the amplitude of the slowroll potential is affected by a multiplicative factor $N$ equal to the dimension of the representation, that is, to the total number of fermions in the theory.

In terms of identifying specific properties of this modified gravity model based on the spectral action, which set it apart from other models, the dependence of the amplitude of the inflation potential on the number of fermions in the particle physics 
sector of the model is so far the most striking feature that distinguishes it from other known slow-roll inflation models.

1.1. Spectral triples. Noncommutative spaces are described, in this context, as a generalization of Riemannian manifolds via the formalism of spectral triples introduced in [10]. An ordinary Riemannian spin manifold $X$ is identified with the spectral triple $\left(C^{\infty}(X), L^{2}(X, S), \not D\right)$, with the algebra of smooth functions acting as multiplication operators on the Hilbert space of square integrable spinors and the Riemannian metric reconstructed from the Dirac operator $\not D$.

More generally, for a noncommutative space, a spectral triple is a similar set $(\mathcal{A}, \mathcal{H}, D)$ consisting of a $*$-algebra represented by bounded operators on a Hilbert space $\mathscr{H}$, and a self-adjoint operator $D$ with compact resolvent acting on $\mathscr{H}$ with a dense domain such that the commutators $[D, a]$ extend to bounded operators on all of $\mathcal{H}$. A finite noncommutative space is one for which the algebra $\mathcal{A}$ and Hilbert space $\mathscr{H}$ are finite dimensional.

A recent powerful reconstruction theorem ([12], see also [29]) shows that commutative spectral triples that satisfy certain natural axioms, related to properties such as orientability, are spectral triples of smooth Riemannian manifolds in the sense mentioned above.

In the models of gravity coupled to matter, the choice of the finite geometry $F=\left(\mathcal{A}_{F}, \mathscr{H}_{F}, D_{F}\right)$ determines the field content of the particle physics model. As shown in [8], the coordinates on the moduli space of possible Dirac operators $D_{F}$ on the finite geometry $\left(\mathcal{A}_{F}, \mathscr{H}_{F}\right)$ specify the Yukawa parameters (Dirac and Majorana masses and mixing angles) for the particles. A classification of the moduli spaces of Dirac operators on general finite geometries was given in [3].

1.2. The spectral action. One obtains then a theory of (modified) gravity coupled to matter by taking as an action functional the spectral action on the noncommutative space $X \times F$, considered as the product of the spectral triples $\left(C^{\infty}(X), L^{2}(X, S), \not D\right)$ and $\left(\mathcal{A}_{F}, \mathscr{H}_{F}, D_{F}\right)$.

The spectral action functional introduced in [5] is a function of the spectrum of the Dirac operator on a spectral triple given by summing over the spectrum with a cutoff function. Namely, the spectral action functional is defined by $\operatorname{Tr}(f(D / \Lambda))$, where $\Lambda$ is an energy scale, $D$ is the Dirac operator of the spectral triple, and $f$ is a smooth approximation to a cutoff function. As shown in [5] this action functional has an asymptotic expansion at high energies $\Lambda$ of the form

$$
\operatorname{Tr}(f(D / \Lambda)) \sim \sum_{k \in \operatorname{DimSp}} f_{k} \Lambda^{k} f|D|^{-k}+f(0) \zeta_{D}(0)+o(1),
$$

where the $f_{k}$ are the momenta $f_{k}=\int_{0}^{\infty} f(v) v^{k-1} d v$ of the test function $f$, for $k$ a non-negative integer in the dimension spectrum of $D$ (the set of poles of the zeta 
functions $\left.\zeta_{a, D}(s)=\operatorname{Tr}\left(a|D|^{-s}\right)\right)$ and the term $f|D|^{-k}$ given by the residue at $k$ of the zeta function $\zeta_{D}(s)$.

These terms in the asymptotic expansion of the spectral action can be computed explicitly: for a suitable choice of the finite geometry spectral triple $\left(\mathcal{A}_{F}, \mathscr{H}_{F}, D_{F}\right)$ as in [8], they recover all the bosonic terms in the Lagrangian of the Standard Model (with additional right-handed neutrinos with Majorana mass terms) and gravitational terms including the Einstein-Hilbert action, a cosmological term, and conformal gravity terms; see also Chapter 1 of [13]. For a different choice of the finite geometry, one can obtain supersymmetric QCD, see [35].

The higher order terms in the spectral action, which appear with coefficients $f_{-2 k}=(-1)^{k} k ! /(2 k) ! f^{(2 k)}(0)$ depending on the derivatives of the test function and involve higher derivative terms in the fields, were considered explicitly recently, in work related to renormalization of the spectral action for gauge theories [36], [37] and also in [6]. In cases where the underlying geometry is very symmetric (space forms) and the Dirac spectrum is explicitly known, it is also possible to obtain explicit nonperturbative computations of the spectral action, computed directly as $\operatorname{Tr}(f(D / \Lambda))$, using Poisson summation formula techniques applied to the Dirac spectrum and its multiplicities; see [6], [20], [21], [34].

1.3. Almost-commutative geometries. It is also natural to consider a generalization of the product geometry $X \times F$, where this type of almost-commutative geometry is generalized to allow for nontrivial fibrations that are only locally, but not globally, products. This means considering almost-commutative geometries that are fibrations over an ordinary manifold $X$, with fiber a finite noncommutative space $F$. A first instance where such topologically non-trivial cases were considered in the context of models of gravity coupled to matter was the Yang-Mills case considered in [1].

In the setting of [1], instead of a product geometry $X \times F$, one considers a noncommutative space obtained as an algebra bundle, namely where the algebra of the full space is isomorphic to sections $\Gamma(X, \mathcal{E})$ of a locally trivial $*$-algebra bundle whose fibers $\varepsilon_{X}$ are isomorphic to a fixed finite-dimensional algebra $\mathcal{A}_{F}$. The spectral triple that replaces the product geometry is of the form $\left(C^{\infty}(X, \mathcal{E}), L^{2}(X, \mathcal{E} \otimes S), D_{\mathcal{E}}\right)$, where the Dirac operator $D_{\mathcal{E}}=c \circ\left(\nabla^{\mathcal{E}} \otimes 1+1 \otimes \nabla^{S}\right)$ is defined using the spin connection and a hermitian connection on the algebra bundle $\mathcal{E}$ (with respect to an inner product obtained using a faithful tracial state $\tau_{x}$ on $\varepsilon_{x}$ ). The spectral triple obtained in this way can be endowed with a compatible grading and real structure and it is described in [1] in terms of an unbounded Kasparov product of KK-cycles. In the Yang-Mills case, where the finite-dimensional algebra is $\mathcal{A}_{F}=M_{N}(\mathbb{C})$, it is shown in [1] that this type of spectral triples describes $\operatorname{PSU}(N)$-gauge theory with a nontrivial principal bundle and the Yang-Mills action functional coupled to gravity is recovered from the asymptotic expansion of the spectral action.

Even more generally, one can define an almost-commutative geometry with base $X$, by a spectral triple of the form $(\mathcal{A}, \mathscr{H}, D)$, where $\mathscr{H}=L^{2}(X, \mathcal{V})$ for $\mathcal{V} \rightarrow X$ a self-adjoint Clifford module bundle, $\mathcal{A}=C^{\infty}(X, \mathcal{E})$ for $\mathcal{E} \rightarrow X$ a unital $*$-algebra 
subbundle of $\operatorname{End}_{\mathrm{Cl}(X)}^{+}(\mathcal{V})$, and $D$ a symmetric Dirac-type operator on $\mathcal{V}$; in this context, the compact Riemannian manifold $X$ is no longer required to be spin. A reconstruction theorem for almost commutative geometries (defined in this more general topologically nontrivial sense was recently obtained in [4] as a consequence of the reconstruction theorem for commutative spectral triples of [12]. There the above concrete definition was shown to be equivalent to an abstract definition of almost-commutative geometry with base a commutative unital $*$-algebra, analogous to the abstract definition of commutative spectral triple.

1.4. Cosmic topology and inflation. The asymptotic expansion of the spectral action naturally provides an action functional for (Euclidean) modified gravity, where in addition to the ordinary Einstein-Hilbert action with cosmological term one also has a topological term (the Euler characteristic) and conformal gravity terms like the Weyl curvature and a conformal coupling of the Higgs field to gravity. It also produces the additional bosonic terms: the action for the Higgs with quartic potential and the Yang-Mills action for the gauge fields. Thus, it is natural to consider the spectral action as a candidate action functional for a modified gravity model and study its consequences for cosmology.

Cosmological implications of the spectral action, based on the asymptotic expansion, were considered in [17], [19], [22], [23], [24], [25]. For recent developments in the case of Robertson-Walker metrics see [7].

In [20] and [21] the nonperturbative spectral action was computed explicitly for the 3-dimensional spherical space forms and the flat 3-dimensional Bieberbach manifolds, via the same type of Poisson summation technique first used in [6] for the sphere case. The spectral action for Bieberbach manifolds was also computed in [28]. In these computations one considers the spectral action as a pure gravity functional (that is, only on the manifold $X$, without the finite geometry $F$ ). It is shown that a perturbation $D^{2}+\phi^{2}$ of the Dirac operator produces in the nonperturbative spectral action a slow-roll potential $V(\phi)$ for the scalar field $\phi$, which can be used as a model for cosmic inflation.

While in [6] the computation of the potential $V(\phi)$ is performed in the model the case of the Higgs field, we do not assume here (nor in [20], [21]) that $\phi$ is necessarily related to the Higgs field and we only treat $D^{2}+\phi^{2}$ as a scalar field perturbation of the Dirac operator. In particular, in the noncommutative geometry models of particle physics the Higgs field arises because of the presence of a nontrivial noncommutative space $F$ and an almost commutative geometry $X \times F$ (the product of the spacetime manifold $X$ and $F$, or more generally a nontrivial fibration as analyzed in [4]). The Higgs field is described geometrically as the inner fluctuations of the Dirac operator in the noncommutative direction $F$. On the other hand, more general fluctuations of the form $D^{2}+\phi^{2}$ are possible also in the pure manifold case (the pure gravity case), in the absence of a noncommutative fiber $F$. These are indeed the cases considered in [20], [21]. It is worth pointing out that if one assumes that the field $\phi$ is related to the Higgs field, then there are very strong constraints coming from the CMB data, as 
recently analyzed in [2], which make a Higgs-based inflation scenario, as predicted by this kind of NCG model, incompatible with the measured value of the top quark mass. However, these constraints do not directly apply to other scalar perturbations $\phi$, not related to the Higgs field.

In this paper we focus on the same topologies considered in [20], [34] and [21]. These include all the most significant candidates for a nontrivial cosmic topology, widely studied in the theoretical cosmology literature (see for example [27], [30], [31]). We refer the reader to [20] and the references therein for a more detailed overview of the specific physical significance of these various topologies.

In [20], [34] and [21] the nonperturbative spectral action is computed for all the spherical space forms $S^{3} / \Gamma$ and for the flat tori and for all the flat Bieberbach 3manifolds (for the latter case see also [28]). These two classes of manifolds provide a complete classification of all the possible homogeneous compact 3-manifolds that are either positively curved or flat, hence they encompass all the possible compact cases of interest to the problem of cosmic topology (see for instance [30], [31]). It is shown in [20], [34] and [21] that the nonperturbative spectral action for spherical space forms is, up to an overall constant factor that depends on the order of the finite group $\Gamma$, the same as that of the sphere $S^{3}$, hence so is the slow-roll potential. Similarly, the spectral action and potentials for the flat Bieberbach manifolds are a multiple of those of the flat torus $T^{3}$. In particular, for each such manifold, although the spectra depend explicitly on the different spin structures, the spectral action does not. These results show that, in a model of gravity based on the spectral action functional, the amplitudes and slow-roll parameters in the power spectra for the scalar and tensor fluctuation would depend on the underlying cosmic topology, and hence constraints on these quantities derived from cosmological data (see [18], [32], [33]) may, in principle, be able to distinguish between different topologies.

Here we discuss a natural question arising from the results of [20] and [21], namely how the presence of the finite geometry $F$ may affect the behavior of the slow-roll inflation potential. As a setting, we consider the case of the spherical space forms $S^{3} / \Gamma$ as the commutative base of an almost commutative geometry in the sense of [4], where the Clifford module bundle $\mathcal{V}$ on $S^{3} / \Gamma$ is the spinor bundle twisted by a flat bundle corresponding to a finite-dimensional representation $\alpha: \Gamma \rightarrow \mathrm{GL}_{N}(\mathbb{C})$ of the group $\Gamma$. As the Dirac operator on the almost commutative geometry we consider the corresponding twisted Dirac operator $D_{\alpha}^{\Gamma}$ on $S^{3} / \Gamma$. From the point of view of the physical model this means that we only focus on the gravity terms and we do not include the part of the Dirac operator $D_{F}$ that describes the matter content and which comes from a finite spectral triple in the fiber direction.

Our main result is that, for any such almost commutative geometry, the spectral action and the associated slow-roll potential only differ from those of the sphere $S^{3}$ by an overall multiplicative amplitude factor equal to $N / \# \Gamma$. Thus, the only modification to the amplitude factor in the power spectra is a correction, which appears uniformly for all topologies, by a multiplicative factor $N$ depending on the fiber of the almost commutative geometry. In terms of the physical model, this $N$ represents the number 
of fermions in the theory. We obtain this by computing the spectral action in its nonperturbative form, as in [6], [20], [21], [34], using the Poisson summation formula technique and the explicit form of the Dirac spectra derived in [9].

More generally, one can consider a finite normal Riemannian cover $\Gamma \rightarrow \widetilde{M} \rightarrow$ $M$ together with a finite-dimensional representation $\alpha: \Gamma \rightarrow U(N)$, and compare the spectral action and slow-roll potential on a $\Gamma$-equivariant almost commutative geometry over $\tilde{M}$, with the spectral action and slow-roll potential on the quotient geometry twisted by $\alpha$, an almost commutative geometry over $M$. Using a nonperturbative heat kernel argument, we find that the spectral action and slow-roll potential over $M$ differ from those over $\tilde{M}$, up to an error of order $O\left(\Lambda^{-\infty}\right)$ as $\Lambda \rightarrow+\infty$, by an overall multiplicative amplitude equal to $N / \# \Gamma$, recovering both our result above for spherical space forms, as well as the relations obtained in [20], [21] and [34]. We also obtain the analogous result for the perturbative spectral action.

1.5. Basic setup. We recall the basic setting, following the notation of [9]. Let $\Gamma \subset \mathrm{SU}(2)$ be a finite group acting by isometries on $S^{3}$, identified with the Lie group $\mathrm{SU}(2)$ with the round metric. The spinor bundle on the spherical form $S^{3} / \Gamma$ is given by $S^{3} \times_{\sigma} \mathbb{C}^{2} \rightarrow S^{3} / \Gamma$, where $\sigma$ is the representation of $\Gamma$ defined by the standard representation of $\mathrm{SU}(2)$ on $\mathbb{C}^{2}$.

A unitary representation $\alpha: \Gamma \rightarrow U(N)$ defines a flat bundle $\mathcal{V}_{\alpha}=S^{3} \times{ }_{\alpha} \mathbb{C}^{N}$ endowed with a canonical flat connection. By twisting the Dirac operator with the flat bundle, one obtains an operator $D_{\alpha}^{\Gamma}$ on the spherical form $S^{3} / \Gamma$ acting on the twisted spinors, that is, on the $\Gamma$-equivariant sections $C^{\infty}\left(S^{3}, \mathbb{C}^{2} \otimes \mathbb{C}^{N}\right)^{\Gamma}$, where $\Gamma$ acts by isometries on $S^{3}$ and by $\sigma \otimes \alpha$ on $\mathbb{C}^{2} \otimes \mathbb{C}^{N}$; these are the sections of the twisted spinor bundle $S^{3} \times_{\sigma \otimes \alpha}\left(\mathbb{C}^{2} \otimes \mathbb{C}^{N}\right) \rightarrow S^{3} / \Gamma$. Thus, $D_{\alpha}^{\Gamma}$ is the restriction of the Dirac operator $D \otimes \operatorname{id}_{\mathbb{C}^{N}}$ to the subspace $C^{\infty}\left(S^{3}, \mathbb{C}^{2} \otimes \mathbb{C}^{N}\right)^{\Gamma} \subset C^{\infty}\left(S^{3}, \mathbb{C}^{2} \otimes \mathbb{C}^{N}\right)$.

This setup gives rise to an almost commutative geometry in the sense of [4], where the twisted Dirac operator $D_{\alpha}^{\Gamma}$ represents the "pure gravity" part of the resulting model of gravity coupled to matter, while the fiber $\mathbb{C}^{N}=\mathscr{H}_{F}$ determines the fermion content of the matter part and can be chosen according to the type of particle physics model one wishes to consider (Standard Model with right-handed neutrinos, supersymmetric QCD, for example, as in [8], [35], or other possibilities). Since we will only be focusing on the gravity terms, we do not need to specify in full the data of the almost commutative geometry, beyond assigning the flat bundle $\mathcal{V}_{\alpha}$ and the twisted Dirac operator $D_{\alpha}^{\Gamma}$, as the additional data would not enter directly in our computations.

We later generalise this above setup as follows. Let $\Gamma \rightarrow \tilde{M} \rightarrow M$ be a finite normal Riemannian covering with $\tilde{M}$ and $M$ compact, let $\widetilde{\mathcal{V}} \rightarrow \tilde{M}$ be a $\Gamma$-equivariant self-adjoint Clifford module bundle, and let $\Gamma$-equivariant symmetric Dirac-type operator $\widetilde{D}$ on $\widetilde{\mathcal{V}}$; let $\alpha: \Gamma \rightarrow U(N)$ be a unitary representation. We can then define a self-adjoint Clifford module bundle $\mathcal{V}_{\alpha}$ on $M$ by $\left(\tilde{\mathcal{V}} \otimes \mathbb{C}^{N}\right) / \Gamma$, and a symmetric Dirac-type operator $D_{\alpha}$ on $\mathcal{V}_{\alpha}$ as the image of $\widetilde{D} \otimes 1_{N}$ acting on $\widetilde{\mathcal{V}} \otimes \mathbb{C}^{N}$. Again, since we will be focusing on the gravity terms, and since our results will allow for 
suitable equivariant perturbations of $\widetilde{D}$, we do not need to specify algebra bundles for $\tilde{\mathcal{V}}$ or for $\mathcal{V}_{\alpha}$.

As it is customary when using the spectral action formalism, the computation in the 4-dimensional case that determines the form of the inflation potential $V(\phi)$ is obtained in Euclidean signature, on a compactification along an $S_{\beta}^{1}$, of size $\beta$. For a detailed discussion of this method and of the significance of the parameter $\beta$, we refer the reader to $\S 3.1$ of [21].

This paper, as well as the previous two parts [20] and [21], are aimed at developing the mathematical aspects of these noncommutative geometry models of cosmology, hence they focus primarily on computing the non-perturbative form of the spectral action for a relevant class of spaces, and showing how one can derive from it a slow-roll inflation potential and the dependence of this potential from parameters of the model (in the present paper, especially the dimension $N$ of the representation). This paper also focuses on comparing two different methods for computing the nonperturbative spectral action, respectively based on the Poisson summation formula and on heat kernel techniques. As such, it is addressed primarily to an audience of mathematicians and mathematical physicists. Ultimately, the success of such models in cosmology will inevitably depend on the more strictly cosmological aspects of this project, namely a direct comparison between the model and the current CMB data. This is indeed the aim of our ongoing collaboration with the cosmologist Elena Pierpaoli and will be addressed elsewhere, as appropriate.

\section{Poisson summation formula}

Following the method developed in [6] and [20], [34], we compute the spectral action of the quotient spaces $S^{3} / \Gamma$ equipped with the twisted Dirac operator corresponding to a finite-dimensional representation $\alpha$ of $\Gamma$ as follows. We define a finite set of polynomials labeled $P_{m}^{+}$and $P_{m}^{-}$that describe the multiplicities of, respectively, the positive and negative eigenvalues of the twisted Dirac operator. More precisely, the index $m$ takes values in the set of residue classes of the integers modulo $c_{\Gamma}$, where $c_{\Gamma}$ is the exponent of the group $\Gamma$, the least common multiple of the orders of the elements in $\Gamma$. For $k \equiv m \bmod c_{\Gamma}, k \geq 1$, and

$$
\lambda=-1 / 2 \pm(k+1),
$$

$P_{m}^{ \pm}(\lambda)$ equals the multiplicity of $\lambda$.

The main technical result we will prove is the following relation between these polynomials:

$$
\sum_{m=1}^{c_{\Gamma}} P_{m}^{+}(u)=\sum_{m=0}^{c_{\Gamma}-1} P_{m}^{-}(u)=\frac{N c_{\Gamma}}{\# \Gamma}\left(u^{2}-\frac{1}{4}\right) .
$$

Since the polynomial on the right-hand side is a multiple of the polynomial for the spectral multiplicities of the Dirac spectrum of the sphere $S^{3}$ (see [6]), we will obtain 
from this the relation between the non-perturbative spectral action of the twisted Dirac operator $D_{\alpha}^{\Gamma}$ on $S^{3} / \Gamma$ and the spectral action on the sphere, see Theorem 2.1 below.

Furthermore, we shall show that the polynomials $P_{m}^{+}(u)$ match up perfectly with the polynomials $P_{m}^{-}(u)$, so that the polynomials $P_{m}^{+}(u)$ alone describe the entire spectrum by allowing the parameter $k$ in eq. (2.1) to run through all of $\mathbb{Z}$. Namely, what we need to show is that

$$
P_{m}^{+}(u)=P_{m^{\prime}}^{-}(u),
$$

where for each $m, m^{\prime}$ is the unique number between 0 and $c_{\Gamma}-1$ such that $m+m^{\prime}+2$ is a multiple of $c_{\Gamma}$, or more precisely,

$$
m^{\prime}= \begin{cases}c_{\Gamma}-2-m, & \text { if } 1 \leq m \leq c_{\Gamma}-2, \\ c_{\Gamma}-1, & \text { if } m=c_{\Gamma}-1, \\ c_{\Gamma}-2 & \text { if } m=c_{\Gamma} .\end{cases}
$$

Define

$$
g_{m}(u)=P_{m}^{+}(u) f(u / \Lambda) .
$$

Now we apply the Poisson summation formula to obtain

$$
\begin{aligned}
\operatorname{Tr}(f(D / \Lambda)) & =\sum_{m} \sum_{l \in \mathbb{Z}} g_{m}\left(1 / 2+c_{\Gamma} l+m+1\right) \\
& =\frac{N}{\# \Gamma} \sum_{m} \widehat{g_{m}}(0)+O\left(\Lambda^{-\infty}\right) \\
& =\frac{N}{\# \Gamma}\left(\int_{\mathbb{R}} u^{2} f(u / \Lambda)-\frac{1}{4} \int_{\mathbb{R}} f(u / \Lambda)\right)+O\left(\Lambda^{-\infty}\right) \\
& =\frac{N}{\# \Gamma}\left(\Lambda^{3} \hat{f}^{(2)}(0)-\frac{1}{4} \Lambda \hat{f}(0)\right)+O\left(\Lambda^{-\infty}\right),
\end{aligned}
$$

and so we have the main result.

Theorem 2.1. Let $\Gamma$ be a finite subgroup of $S^{3}$, and let $\alpha$ be a $N$-dimensional representation of $\Gamma$. Then the spectral action of $S^{3} / \Gamma$ equipped with the twisted Dirac operator is

$$
\operatorname{Tr} f(D / \Lambda)=\frac{N}{\# \Gamma}\left(\Lambda^{3} \hat{f}^{(2)}(0)-\frac{1}{4} \Lambda \hat{f}(0)\right)+O\left(\Lambda^{-\infty}\right),
$$

where $\hat{f}^{(2)}$ denotes the Fourier transform of $u^{2} f(u)$.

Similar computations of the spectral action have also been performed in [20], [21], and [34]. In the sequel we describe how to obtain eq. (2.2), by explicitly analyzing the cases of the various spherical space forms: lens spaces, dicyclic group, and binary tetrahedral, octahedral, and icosahedral groups. In all cases we compute explicitly the polynomials of the spectral multiplicities and check that (2.2) is satisfied. Our calculations are based on a result of Cisneros-Molina, [9], on the explicit form of the Dirac spectra of the twisted Dirac operators $D_{\alpha}^{\Gamma}$, which we recall here below. 
2.1. Twisted Dirac spectra of spherical space forms. The spectra of the twisted Dirac operators on the quotient spaces are derived in [9]. Let us recall the notation and the main results.

Let $E_{k}$ denote the $k+1$-dimensional irreducible representation of SU(2) on the space of homogeneous complex polynomials in two variables of degree $k$. By the Peter-Weyl theorem, one can decompose $C^{\infty}\left(S^{3}, \mathbb{C}\right)=\bigoplus_{k} E_{k} \otimes E_{k}^{*}$ as a sum of irreducible representations of SU(2). This gives that, on $C^{\infty}\left(S^{3}, \mathbb{C}^{2} \otimes\right.$ $\left.\mathbb{C}^{N}\right)=\bigoplus_{k} E_{k} \otimes E_{k}^{*} \otimes \mathbb{C}^{2} \otimes \mathbb{C}^{N}$, the Dirac operator $D \otimes \mathrm{id}_{\mathbb{C}^{N}}$ decomposes as $\bigoplus_{k} \mathrm{id}_{E_{k}} \otimes D_{k} \otimes \mathrm{id}_{\mathbb{C}^{N}}$, with $D_{k}: E_{k}^{*} \otimes \mathbb{C}^{2} \rightarrow E_{k}^{*} \otimes \mathbb{C}^{2}$. Upon identifying $C^{\infty}\left(S^{3}, \mathbb{C}^{2} \otimes \mathbb{C}^{N}\right)^{\Gamma}=\bigoplus_{k} E_{k} \otimes \operatorname{Hom}_{\Gamma}\left(E_{k}, \mathbb{C}^{2} \otimes \mathbb{C}^{N}\right)$, one sees that, as shown in [9], the multiplicities of the spectrum of the twisted Dirac operator $D_{\alpha}^{\Gamma}$ are given by the dimensions $\operatorname{dim}_{\mathbb{C}} \operatorname{Hom}_{\Gamma}\left(E_{k}, \mathbb{C}^{2} \otimes \mathbb{C}^{N}\right)$, which in turn can be expressed in terms of the pairing of the characters of the corresponding $\Gamma$-representation, that is, as $\left\langle\chi_{E_{k}}, \chi_{\sigma \otimes \alpha}\right\rangle_{\Gamma}$. One then obtains the following:

Theorem 2.2 (Cisneros-Molina, [9]). Let $\alpha: \Gamma \rightarrow G L_{N}(\mathbb{C})$ be a representation of $\Gamma$. Then the eigenvalues of the twisted Dirac operator $D_{\alpha}^{\Gamma}$ on $S^{3} / \Gamma$ are

$$
\begin{aligned}
& -\frac{1}{2}-(k+1) \text { with multiplicity }\left\langle\chi_{\left.E_{k+1}, \chi_{\alpha}\right\rangle_{\Gamma}(k+1)} \text { if } k \geq 0,\right. \\
& -\frac{1}{2}+(k+1) \text { with multiplicity }\left\langle\chi_{E_{k-1}}, \chi_{\alpha}\right\rangle_{\Gamma}(k+1) \quad \text { if } k \geq 1 .
\end{aligned}
$$

Proposition 2.3 (Cisneros-Molina, [9]). Let $k=c_{\Gamma} l+m$ with $0 \leq m<c_{\Gamma}$.

(1) If $-1 \in \Gamma$, then

$$
\left\langle\chi_{E_{k}}, \chi_{\alpha}\right\rangle_{\Gamma}= \begin{cases}\frac{c_{\Gamma} l}{\# \Gamma}\left(\chi_{\alpha}(1)+\chi_{\alpha}(-1)\right)+\left\langle\chi_{E_{m}}, \chi_{\alpha}\right\rangle_{\Gamma} & \text { if } k \text { is even }, \\ \frac{c_{\Gamma} l}{\# \Gamma}\left(\chi_{\alpha}(1)-\chi_{\alpha}(-1)\right)+\left\langle\chi_{E_{m}}, \chi_{\alpha}\right\rangle_{\Gamma} & \text { if } k \text { is odd. }\end{cases}
$$

(2) If $-1 \notin \Gamma$, then

$$
\left\langle\chi_{E_{k}}, \chi_{\alpha}\right\rangle_{\Gamma}=\frac{N c_{\Gamma} l}{\# \Gamma}+\left\langle\chi_{E_{m}}, \chi_{\alpha}\right\rangle_{\Gamma}
$$

2.2. Lens spaces, odd order. In this section we consider $\Gamma=\mathbb{Z}_{n}$, where $n$ is odd. When $n$ is odd, $-1 \notin \Gamma$, which affects the expression for the character inner products in Proposition 2.3.

For $m \in\{1, \ldots, n\}$, we introduce the polynomials,

$$
P_{m}^{+}(u)=\frac{N}{n} u^{2}+\left(\beta_{m}^{\alpha}-\frac{m N}{n}\right) u+\frac{\beta_{m}^{\alpha}}{2}-\frac{m N}{2 n}-\frac{N}{4 n},
$$

where

$$
\beta_{m}^{\alpha}=\left\langle\chi_{E_{m-1}}, \chi_{\alpha}\right\rangle_{\Gamma}
$$

and $m$ takes on values in $\{1,2, \ldots, n\}$. 
Using Theorem 2.2 and Proposition 2.3, it is easy to see that the polynomials $P_{m}^{+}(u)$ describe the spectrum on the positive side of the real line, i.e., $P_{m}^{+}(\lambda)$ equals the multiplicity of

$$
\lambda=-1 / 2+(k+1), \quad k \geq 1,
$$

whenever $k \equiv m \bmod n$.

For the negative eigenvalues, the multiplicities are described by the polynomials

$$
P_{m}^{-}(u)=\frac{N}{n} u^{2}+\left(\frac{2 N}{n}+\frac{m N}{n}-\gamma_{m}^{\alpha}\right) u+\frac{3 N}{4 n}+\frac{m N}{2 n}-\frac{\gamma_{m}^{\alpha}}{2},
$$

$m \in\{0,1, \ldots n-1\}$, i.e., $P_{m}^{-}(\lambda)$ is equal to the multiplicity of the eigenvalue

$$
\lambda=-1 / 2-(k+1), \quad k \geq 0,
$$

whenever $k \equiv m \bmod n$; here $\gamma_{m}^{\alpha}$ is defined by

$$
\gamma_{m}^{\alpha}=\left\langle\chi_{E_{m+1}}, \chi_{\alpha}\right\rangle_{\Gamma}
$$

Let us denote the irreducible representations of $\mathbb{Z}_{n}$ by $\chi_{t}$, which sends the generator to $\exp \left(\frac{2 \pi i t}{N}\right)$ for $t$ a residue class of integers modulo $n$.

For the sake of computation, we take $\mathbb{Z}_{n}$ to be the group generated by

$$
B=\left[\begin{array}{cc}
e^{\frac{2 \pi i}{n}} & 0 \\
0 & e^{-\frac{2 \pi i}{n}}
\end{array}\right]
$$

Then in the representation $E_{k}, B$ acts on the basis polynomials $P_{j}\left(z_{1}, z_{2}\right), j \in$ $\{0,1, \ldots k\}$ as follows.

$$
\begin{aligned}
B \cdot P_{j}\left(z_{1}, z_{2}\right) & =P_{j}\left(\left(z_{1}, z_{2}\right) B\right) \\
& =P_{j}\left(e^{\frac{2 \pi i}{n}} z_{1}, e^{-\frac{2 \pi i}{n}} z_{2}\right) \\
& =\left(e^{\frac{2 \pi i}{n}} z_{1}\right)^{k-j}\left(e^{-\frac{2 \pi i}{n}} z_{2}\right)^{j} \\
& =e^{\frac{2 \pi i}{n}(k-2 j)} P_{j}\left(z_{1}, z_{2}\right) .
\end{aligned}
$$

Hence, $B$ is represented by a diagonal matrix with respect to this basis, and we have

Proposition 2.4. The irreducible characters $\chi_{E_{k}}$ of the irreducible representations of $\mathrm{SU}(2)$ restricted to $\mathbb{Z}_{n}$, $n$ odd, are decomposed into the irreducible characters $\chi_{[t]}$ of $\mathbb{Z}_{n}$ by the equation

$$
\chi_{E_{k}}=\sum_{j=0}^{j=k} \chi[k-2 j] .
$$

Here, $[t]$ denotes the number from 0 to $n-1$ to which $t$ is equivalent mod $n$. 
In the case where $-1 \notin \Gamma$, that is to say, when $\Gamma=\mathbb{Z}_{n}$ where $n$ is odd, by equating coefficients of the quadratic polynomials $P_{m}^{+}$and $P_{m^{\prime}}^{-}$, the condition (2.3) is replaced by one that may be simply checked.

Lemma 2.5. Let $\Gamma$ be any finite subgroup of $\mathrm{SU}(2)$ such that $-1 \notin \Gamma$. Then condition (2.3) is equivalent to the condition

$$
\beta_{m}^{\alpha}+\gamma_{m^{\prime}}^{\alpha}= \begin{cases}\chi_{\alpha}(1), & \text { if } 1 \leq m \leq c_{\Gamma}-2, \\ 2 \chi_{\alpha}(1), & \text { if } m=c_{\Gamma}-1, c_{\Gamma},\end{cases}
$$

where $\alpha$ is an irreducible representation of $\Gamma$. Furthermore this condition holds in all cases.

Using Proposition 2.4, it is a simple combinatorial matter to see that

$$
\sum_{m=1}^{n}\left\langle\chi_{E_{m-1}}, \chi_{\alpha}\right\rangle_{\Gamma}=N \frac{n+1}{2},
$$

for any representation $\alpha$ of $\mathbb{Z}_{n}$

For the argument to go through, one also needs to check the special case

$$
P_{c_{\Gamma}}^{+}(1 / 2)=0 .
$$

By direct evaluation one can check that this indeed holds.

For the negative side, we see that

$$
\sum_{m=1}^{n}\left\langle\chi_{E_{m+1}}, \chi_{\alpha}\right\rangle_{\Gamma}=N \frac{n+3}{2}
$$

for any representation $\alpha$ of $\mathbb{Z}_{n}$. Thus we obtain:

Proposition 2.6. Let $\Gamma$ be cyclic with $\# \Gamma$ odd, and let $\alpha$ be a $N$-dimensional representation of $\Gamma$. Then

$$
\sum_{m=1}^{n} P_{m}^{+}(u)=\sum_{m=0}^{n-1} P_{m}^{-}(u)=N u^{2}-\frac{N}{4} .
$$

Note that in the statement of Theorem 2.2, the first line holds even if we take $k=$ -1 since the multiplicity for this value evaluates to zero. Therefore, we automatically have

$$
P_{c_{\Gamma}-1}^{-}(-1 / 2)=0,
$$

which we still needed to check. 
2.3. Lens spaces, even order. When $n$ is even, we have $-1 \in \mathbb{Z}_{n}$. When $-1 \in \Gamma$, from Theorems 2.2 and 2.3 it follows that the multiplicity of the eigenvalue

$$
\lambda=1 / 2+l c_{\Gamma}+m, \quad l \in \mathbb{N},
$$

is given by

$$
\begin{aligned}
P_{m}^{+}(u)= & \frac{1}{\# \Gamma}\left(\chi_{\alpha}(1)+(-1)^{m+1} \chi_{\alpha}(-1)\right) u^{2} \\
& +\left(\beta_{m}^{\alpha}-\frac{1}{\# \Gamma}\left(m\left(\chi_{\alpha}(1)+(-1)^{m+1} \chi_{\alpha}(-1)\right)\right) u\right. \\
& +\frac{1}{2} \beta_{m}^{\alpha}-\frac{1}{4 \# \Gamma}\left(\chi_{\alpha}(1)+(-1)^{m+1} \chi_{\alpha}(-1)\right) \\
& \left.-\frac{1}{2 \# \Gamma} m\left(\chi_{\alpha}(1)+(-1)^{m+1} \chi_{\alpha}(-1)\right)\right),
\end{aligned}
$$

$m \in\left\{1,2, \ldots, c_{\Gamma}\right\}$. The one case that is not clear is that of $\lambda=1 / 2$, which is not an eigenvalue of the twisted Dirac operator. It is not clear from Theorems 2.2 and 2.3 that

$$
P_{c_{\Gamma}}^{+}(1 / 2)=0,
$$

and this needs to hold in order for the argument using the Poisson summation formula to go through. However, by evaluating eq. (2.4) we see that one needs to check that

$$
\left\langle\chi_{E_{c^{-1}}}, \chi_{\alpha}\right\rangle=\frac{c_{\Gamma}}{\# \Gamma}\left(\chi_{\alpha}(1)+(-1)^{c_{\Gamma}+1} \chi_{\alpha}(-1)\right),
$$

so that eq. (2.4) indeed holds for each subgroup $\Gamma$ and irreducible representation $\alpha$.

Proposition 2.7. For any subgroup $\Gamma \subset S^{3}$ of even order, the sum of the polynomials $P_{m}^{+}$is

$$
\begin{aligned}
\sum_{m=1}^{c_{\Gamma}} P_{m}^{+}(u)= & \frac{c_{\Gamma}}{\# \Gamma} \chi_{\alpha}(1) u^{2}+\left(-\frac{c_{\Gamma}^{2} \chi_{\alpha}(1)}{2 \# \Gamma}-\frac{c_{\Gamma}\left(\chi_{\alpha}(1)-\chi_{\alpha}(-1)\right)}{2 \# \Gamma}+\sum_{m=1}^{c_{\Gamma}} \beta_{m}^{\alpha}\right) u \\
& -\frac{c_{\Gamma} \chi_{\alpha}(1)}{2 \# \Gamma}-\frac{c_{\Gamma}^{2} \chi_{\alpha}(1)}{4 \# \Gamma}+\frac{c_{\Gamma}}{4 \# \Gamma} \chi_{\alpha}(-1)+\frac{1}{2} \sum_{m=1}^{c_{\Gamma}} \beta_{m}^{\alpha} .
\end{aligned}
$$

Since the coefficients of the polynomial are additive with respect to direct sum, it suffices to consider only irreducible representations.

In the case of lens spaces, $c_{\Gamma}=\# \Gamma$ and $\chi_{t}(-1)=(-1)^{t}$. As a matter of counting, the following holds:

\section{Proposition 2.8.}

$$
\sum_{m=1}^{c_{\Gamma}} \beta_{m}^{t}= \begin{cases}\frac{n+2}{2} & \text { ift is even }, \\ \frac{n}{2} & \text { ift } \text { is odd } .\end{cases}
$$


Putting this all into the expression of Proposition 2.7, we have, for an $N$-dimensional representation $\alpha$,

$$
\sum_{m=1}^{c_{\Gamma}} P_{m}^{+}(u)=N\left(u^{2}-\frac{1}{4}\right) .
$$

The negative eigenvalues are described by the polynomials

$$
\begin{aligned}
P_{m}^{-}(u)= & \frac{1}{\# \Gamma}\left(\chi_{\alpha}(1)+(-1)^{m+1} \chi_{\alpha}(-1)\right) u^{2} \\
& +\left(\frac{2+m}{\# \Gamma}\left(\chi_{\alpha}(1)+(-1)^{m+1} \chi_{\alpha}(-1)\right)-\gamma_{m}^{\alpha}\right) u \\
& \cdot \frac{3+2 m}{4 \# \Gamma}\left(\chi_{\alpha}(1)+(-1)^{m+1} \chi_{\alpha}(-1)\right)-\frac{1}{2} \gamma_{m}^{\alpha}
\end{aligned}
$$

for $m \in\left\{0,1, \ldots c_{\Gamma}-1\right\}$. So we have the following result.

Proposition 2.9. For any subgroup $\Gamma \subset S^{3}$ of even order, the sum of the polynomials $P_{m}^{-}$is

$$
\begin{aligned}
\sum_{m=1}^{c_{\Gamma}} P_{m}^{-}(u)= & \frac{c_{\Gamma}}{\# \Gamma} \chi_{\alpha}(1) u^{2} \\
& +\left(\frac{\chi_{\alpha}(1) c_{\Gamma}^{2}}{2 \# \Gamma}+\frac{3 \chi_{\alpha}(1) c_{\Gamma}}{2 \# \Gamma}+\frac{\chi_{\alpha}(-1) c_{\Gamma}}{2 \# \Gamma}-\sum_{m=0}^{c_{\Gamma}^{-1}} \gamma_{m}^{\alpha}\right) u \\
& +\frac{\chi_{\alpha}(1) c_{\Gamma}}{2 \# \Gamma}+\frac{\chi_{\alpha}(1) c_{\Gamma}^{2}}{4 \# \Gamma}+\frac{\chi_{\alpha}(-1) c_{\Gamma}}{4 \# \Gamma}-\frac{1}{2} \sum_{m=0}^{c_{\Gamma}^{-1}} \gamma_{m}^{\alpha} .
\end{aligned}
$$

By counting, one can see that

$$
\sum_{m=0}^{c_{\Gamma}-1} \gamma_{m}^{t}= \begin{cases}\frac{n+4}{2} & \text { if } t \text { is even } \\ \frac{n+2}{2} & \text { if } t \text { is odd }\end{cases}
$$

To complete the computation of the spectral action one still needs to verify the condition (2.3). We have the following lemma, obtained by equating the coefficients of $P_{m}^{+}$and $P_{m^{\prime}}^{-}$, that covers the cases of the binary tetrahedral, octahedral and icosahedral groups as well.

Lemma 2.10. Let $\Gamma$ be any finite subgroup of $\mathrm{SU}(2)$ such that $-1 \in \Gamma$. Then the condition (2.3) is equivalent to the condition

$$
\beta_{m}^{\alpha}+\gamma_{m^{\prime}}^{\alpha}= \begin{cases}\chi_{\alpha}(1)\left(\chi_{\alpha}(1)+(-1)^{m+1} \chi_{\alpha}(-1)\right) & \text { if } 1 \leq m \leq c_{\Gamma}-2, \\ 2 \chi_{\alpha}(1)\left(\chi_{\alpha}(1)+\chi_{\alpha}(-1)\right) & \text { if } m=c_{\Gamma}-1, \\ 2 \chi_{\alpha}(1)\left(\chi_{\alpha}(1)-\chi_{\alpha}(-1)\right) & \text { if } m=c_{\Gamma},\end{cases}
$$


where $\alpha$ is an irreducible representation of $\Gamma$. Furthermore this condition holds in all cases.

2.4. Dicyclic group. The character table for the dicyclic group of order $4 r$ is, for $r$ odd,

\begin{tabular}{|c|c|c|c|c|c|}
\hline Class & $1_{+}$ & $1_{-}$ & $2_{l}$ & $r_{0}$ & $r_{1}$ \\
\hline$\psi_{t}$ & 2 & $2(-1)^{t}$ & $\zeta_{2 r}^{l t}+\zeta_{2 r}^{-l t}$ & 0 & 0 \\
\hline$\chi_{1}$ & 1 & 1 & 1 & 1 & 1 \\
\hline$\chi_{2}$ & 1 & -1 & $(-1)^{l}$ & $i$ & $-i$ \\
\hline$\chi_{3}$ & 1 & 1 & 1 & -1 & -1 \\
\hline$\chi_{4}$ & 1 & -1 & $(-1)^{l}$ & $-i$ & $i$, \\
\hline
\end{tabular}

and for $r$ even,

\begin{tabular}{|c|c|c|c|c|c|}
\hline Class & $1_{+}$ & $1_{-}$ & $2_{l}$ & $r_{0}$ & $r_{1}$ \\
\hline$\psi_{t}$ & 2 & $2(-1)^{t}$ & $\zeta_{2 r}^{l t}+\zeta_{2 r}^{-l t}$ & 0 & 0 \\
\hline$\chi_{1}$ & 1 & 1 & 1 & 1 & 1 \\
\hline$\chi_{2}$ & 1 & -1 & $(-1)^{l}$ & $i$ & $-i$ \\
\hline$\chi_{3}$ & 1 & 1 & 1 & -1 & -1 \\
\hline$\chi_{4}$ & 1 & -1 & $(-1)^{l}$ & $-i$ & $i$ \\
\hline
\end{tabular}

Here $\zeta_{2 r}=e^{\frac{\pi i}{r}}, 1 \leq t \leq r-1,1 \leq l \leq r-1$. The notation for the different conjugacy classes can be understood as follows. The number indicates the order of the conjugacy class, while a sign in the subscript indicates the sign of the traces of the elements in the conjugacy class as elements of SU(2).

For the dicyclic group of order $4 r$, the exponent of the group is

$$
c_{\Gamma}= \begin{cases}2 r & \text { if } r \text { is even } \\ 4 r & \text { if } r \text { is odd. }\end{cases}
$$

One can decompose the characters $\chi_{E_{k}}$ into the irreducible characters by inspection, and with some counting obtain the following propositions.

Proposition 2.11. Let $\Gamma$ be the dicyclic group of order $4 r$, where $r$ is even. Then

$$
\begin{aligned}
& \sum_{m=1}^{c_{\Gamma}} \beta_{m}^{\alpha}= \begin{cases}\frac{r}{2} & \text { if } \chi_{\alpha} \in\left\{\chi_{1}, \chi_{2}, \chi_{3}, \chi_{4}\right\} \\
r & \text { if } \chi_{\alpha}=\psi_{t}, \text { t is even } \\
r+1 & \text { if } \chi_{\alpha}=\psi_{t}, t \text { is odd }\end{cases} \\
& \sum_{m=0}^{c_{\Gamma}^{-1}} \gamma_{m}^{\alpha}= \begin{cases}\frac{r}{2}+1 & \text { if } \chi_{\alpha} \in\left\{\chi_{1}, \chi_{2}, \chi_{3}, \chi_{4}\right\} \\
r+2 & \text { if } \chi_{\alpha}=\psi_{t}, t \text { is even } \\
r+1 & \text { if } \chi_{\alpha}=\psi_{t}, t \text { is odd }\end{cases}
\end{aligned}
$$


Proposition 2.12. Let $\Gamma$ be the dicyclic group of order $4 r$, where $r$ is odd. Then

$$
\begin{aligned}
& \sum_{m=1}^{c_{\Gamma}} \beta_{m}^{\alpha}= \begin{cases}2 r & \text { if } \chi_{\alpha} \in\left\{\chi_{1}, \chi_{3}\right\}, \\
2 r+1 & \text { if } \chi_{\alpha} \in\left\{\chi_{2}, \chi_{4}\right\}, \\
4 r & \text { if } \chi_{\alpha}=\psi_{t}, t \text { is even, } \\
4 r+2 & \text { if } \chi_{\alpha}=\psi_{t}, t \text { is odd; }\end{cases} \\
& \sum_{m=0}^{c_{\Gamma}^{-1}} \gamma_{m}^{\alpha}= \begin{cases}2 r+2 & \text { if } \chi_{\alpha} \in\left\{\chi_{1}, \chi_{3}\right\}, \\
2 r+1 & \text { if } \chi_{\alpha} \in\left\{\chi_{2}, \chi_{4}\right\}, \\
4 r+4 & \text { if } \chi_{\alpha}=\psi_{t}, t \text { is even, } \\
4 r+2 & \text { if } \chi_{\alpha}=\psi_{t}, t \text { is odd. }\end{cases}
\end{aligned}
$$

2.5. Binary tetrahedral group. The binary tetrahedral group has order 24 and exponent 12 . The character table of the binary tetrahedral group is as follows:

\begin{tabular}{|c|c|c|c|c|c|c|c|}
\hline Class & $1_{+}$ & $1_{-}$ & $4_{a+}$ & $4_{b+}$ & $4_{a-}$ & $4_{b-}$ & 6 \\
\hline Order & 1 & 2 & 6 & 6 & 3 & 3 & 4 \\
\hline$\chi_{1}$ & 1 & 1 & 1 & 1 & 1 & 1 & 1 \\
\hline$\chi_{2}$ & 1 & 1 & $\omega^{2}$ & $\omega$ & $\omega$ & $\omega^{2}$ & 1 \\
\hline$\chi_{3}$ & 1 & 1 & $\omega$ & $\omega^{2}$ & $\omega^{2}$ & $\omega$ & 1 \\
\hline$\chi_{4}$ & 2 & -2 & 1 & 1 & -1 & -1 & 0 \\
\hline$\chi_{5}$ & 2 & -2 & $\omega^{2}$ & $\omega$ & $-\omega$ & $-\omega^{2}$ & 0 \\
\hline$\chi_{6}$ & 2 & -2 & $\omega$ & $\omega^{2}$ & $-\omega^{2}$ & $-\omega$ & 0 \\
\hline$\chi_{7}$ & 3 & 3 & 0 & 0 & 0 & 0 & -1 \\
\hline
\end{tabular}

Here, $\omega=e^{\frac{2 \pi i}{3}}$.

For the remaining three groups, we can use matrix algebra to decompose the characters $\chi_{E_{k}}$.

Let $\chi_{j}, x_{j}, j=1,2 \ldots, d$, denote the irreducible characters and representatives of the conjugacy classes of the group $\Gamma$, respectively. Then, since every character decomposes uniquely into the irreducible ones, we have a unique expression for $\chi_{E_{k}}$ as the linear combination

$$
\chi_{E_{k}}=\sum_{j=0}^{d} c_{j}^{k} \chi_{j} .
$$

If we let $b=\left(b_{j}\right) j=1, \ldots, d$ be the column with $b_{j}=\chi_{E_{k}}\left(x_{j}\right)$, and let $A=\left(a_{i j}\right)$ be the $d \times d$ matrix where $a_{i j}=\chi_{j}\left(x_{i}\right)$ and let $c=\left(c_{j}^{k}\right) j=1, \ldots d$ be another column, then we have

$$
b=A c
$$

but $A$ is necessarily invertible by the uniqueness of the coefficient column $c$, and so $c$ is given by

$$
c=A^{-1} b \text {. }
$$

By this method, we obtain the following proposition. 
Proposition 2.13. Let $\Gamma$ be the binary tetrahedral group. Then

$$
\begin{aligned}
& \sum_{m=1}^{c_{\Gamma}} \beta_{m}^{\alpha}= \begin{cases}3, & \text { if } \chi_{\alpha} \in\left\{\chi_{1}, \chi_{2}, \chi_{3}\right\}, \\
7, & \text { if } \chi_{\alpha} \in\left\{\chi_{4}, \chi_{5}, \chi_{6}\right\}, \\
9, & \text { if } \chi_{\alpha}=\chi_{7} ;\end{cases} \\
& \sum_{m=0}^{c_{\Gamma}^{-1}} \gamma_{m}^{\alpha}= \begin{cases}4, & \text { if } \chi_{\alpha} \in\left\{\chi_{1}, \chi_{2}, \chi_{3}\right\}, \\
7, & \text { if } \chi_{\alpha} \in\left\{\chi_{4}, \chi_{5}, \chi_{6}\right\}, \\
12, & \text { if } \chi_{\alpha}=\chi_{7} .\end{cases}
\end{aligned}
$$

2.6. Binary octahedral group. The binary octahedral group has order 48 and exponent 24 . The character table of the binary octahedral group is as follows:

\begin{tabular}{|c|c|c|c|c|c|c|c|c|}
\hline Class & $1_{+}$ & $1_{-}$ & $6_{+}$ & $6_{0}$ & $6_{-}$ & $8_{+}$ & $8_{-}$ & 12 \\
\hline Order & 1 & 2 & 8 & 4 & 8 & 6 & 3 & 4 \\
\hline$\chi_{1}$ & 1 & 1 & 1 & 1 & 1 & 1 & 1 & 1 \\
\hline$\chi_{2}$ & 1 & 1 & -1 & 1 & -1 & 1 & 1 & -1 \\
\hline$\chi_{3}$ & 2 & 2 & 0 & 2 & 0 & -1 & -1 & 0 \\
\hline$\chi_{4}$ & 2 & -2 & $\sqrt{2}$ & 0 & $-\sqrt{2}$ & 1 & -1 & 0 \\
\hline$\chi_{5}$ & 2 & -2 & $-\sqrt{2}$ & 0 & $\sqrt{2}$ & 1 & -1 & 0 \\
\hline$\chi_{6}$ & 3 & 3 & -1 & -1 & -1 & 0 & 0 & 1 \\
\hline$\chi_{7}$ & 3 & 3 & 1 & -1 & 1 & 0 & 0 & -1 \\
\hline$\chi_{8}$ & 4 & -4 & 0 & 0 & 0 & -1 & 1 & 0 \\
\hline
\end{tabular}

Proposition 2.14. Let $\Gamma$ be the binary octahedral group. Then

$$
\begin{aligned}
& \sum_{m=1}^{c_{\Gamma}} \beta_{m}^{\alpha}= \begin{cases}6, & \text { if } \chi_{\alpha} \in\left\{\chi_{1}, \chi_{2}\right\} \\
12, & \text { if } \chi_{\alpha}=\chi_{3}, \\
13, & \text { if } \chi_{\alpha} \in\left\{\chi_{4}, \chi_{5}\right\}, \\
18, & \text { if } \chi_{\alpha} \in\left\{\chi_{6}, \chi_{7}\right\} \\
26, & \text { if } \chi_{\alpha}=\chi_{8}\end{cases} \\
& \sum_{m=0}^{c_{\Gamma}-1} \gamma_{m}^{\alpha}= \begin{cases}7, & \text { if } \chi_{\alpha} \in\left\{\chi_{1}, \chi_{2}\right\}, \\
14, & \text { if } \chi_{\alpha}=\chi_{3}, \\
13, & \text { if } \chi_{\alpha} \in\left\{\chi_{4}, \chi_{5}\right\}, \\
21, & \text { if } \chi_{\alpha} \in\left\{\chi_{6}, \chi_{7}\right\} \\
26, & \text { if } \chi_{\alpha}=\chi_{8} .\end{cases}
\end{aligned}
$$

2.7. Binary icosahedral group. The binary icosahedral group has order 120 and exponent 60 . The character table of the binary icosahedral group is as follows: 


\begin{tabular}{|c|c|c|c|c|c|c|c|c|c|}
\hline Class & $1_{+}$ & $1_{-}$ & 30 & $20_{+}$ & $20_{-}$ & $12_{a+}$ & $12_{b+}$ & $12_{a-}$ & $12_{b-}$ \\
\hline Order & 1 & 2 & 4 & 6 & 3 & 10 & 5 & 5 & 10 \\
\hline$\chi_{1}$ & 1 & 1 & 1 & 1 & 1 & 1 & 1 & 1 & 1 \\
\hline$\chi_{2}$ & 2 & -2 & 0 & 1 & -1 & $\mu$ & $v$ & $-\mu$ & $-v$ \\
\hline$\chi_{3}$ & 2 & -2 & 0 & 1 & -1 & $-v$ & $-\mu$ & $v$ & $\mu$ \\
\hline$\chi_{4}$ & 3 & 3 & -1 & 0 & 0 & $-v$ & $\mu$ & $-v$ & $\mu$ \\
\hline$\chi_{5}$ & 3 & 3 & -1 & 0 & 0 & $\mu$ & $-v$ & $\mu$ & $-v$ \\
\hline$\chi_{6}$ & 4 & 4 & 0 & 1 & 1 & -1 & -1 & -1 & -1 \\
\hline$\chi_{7}$ & 4 & -4 & 0 & -1 & 1 & 1 & -1 & -1 & 1 \\
\hline$\chi_{8}$ & 5 & 5 & 1 & -1 & -1 & 0 & 0 & 0 & 0 \\
\hline$\chi_{9}$ & 6 & -6 & 0 & 0 & 0 & -1 & 1 & 1 & -1 \\
\hline
\end{tabular}

Here, $\mu=\frac{\sqrt{5}+1}{2}$, and $v=\frac{\sqrt{5}-1}{2}$.

Proposition 2.15. Let $\Gamma$ be the binary icosahedral group. Then

$$
\begin{gathered}
\sum_{m=1}^{c_{\Gamma}} \beta_{m}^{\alpha}= \begin{cases}15, & \text { if } \chi_{\alpha}=\chi_{1}, \\
31, & \text { if } \chi_{\alpha} \in\left\{\chi_{2}, \chi_{3}\right\}, \\
45, & \text { if } \chi_{\alpha} \in\left\{\chi_{4}, \chi_{5}\right\}, \\
60, & \text { if } \chi_{\alpha}=\chi_{6}, \\
62, & \text { if } \chi_{\alpha}=\chi_{7}, \\
75, & \text { if } \chi_{\alpha}=\chi_{8}, \\
93, & \text { if } \chi_{\alpha}=\chi_{9} ;\end{cases} \\
\sum_{m=0}^{c_{\Gamma}-1} \gamma_{m}^{\alpha}= \begin{cases}16, & \text { if } \chi_{\alpha}=\chi_{1}, \\
31, & \text { if } \chi_{\alpha} \in\left\{\chi_{2}, \chi_{3}\right\}, \\
48, & \text { if } \chi_{\alpha} \in\left\{\chi_{4}, \chi_{5}\right\}, \\
64, & \text { if } \chi_{\alpha}=\chi_{6}, \\
62, & \text { if } \chi_{\alpha}=\chi_{7}, \\
80, & \text { if } \chi_{\alpha}=\chi_{8}, \\
93, & \text { if } \chi_{\alpha}=\chi_{9} .\end{cases}
\end{gathered}
$$

2.8. Sums of polynomials. If we input the results of Propositions $2.8,2.11,2.12$, 2.13, 2.14, 2.15 into Propositions 2.7, 2.9, and also recall Proposition 2.6, we obtain the following.

Proposition 2.16. Let $\Gamma$ be any finite subgroup of $\mathrm{SU}(2)$ and let $\alpha$ be an $N$ dimensional representation of $\Gamma$. Then the sums of the polynomials $P_{m}^{+}$and $P_{m}^{-}$ are given by

$$
\sum_{m=1}^{c_{\Gamma}} P_{m}^{+}(u)=\sum_{m=0}^{c_{\Gamma}^{-1}} P_{m}^{-}(u)=\frac{N c_{\Gamma}}{\# \Gamma}\left(u^{2}-\frac{1}{4}\right)
$$




\section{A heat kernel argument}

It may at first seem surprising that, in the above calculation, using the Poisson summation formula and the explicit Dirac spectra, although the spectra themselves depend in a subtle way upon the representation-theoretic data of the unitary representation $\alpha: \Gamma \rightarrow U(N)$, through the pairing of the characters of representations, the resulting spectral action only depends upon the dimension $N$ of the representation, the order of $\Gamma$ and the spectral action on $S^{3}$.

This phenomenon is parallel to the similar observation in the Poisson formula computation of the spectral action for the spherical space forms and the flat Bieberbach manifolds in the untwisted case [20], [21], [34], where one finds that, although the Dirac spectra are different for different spin structures, the resulting spectral action depends only on the order \# $\Gamma$ of the finite group and the spectral action on $S^{3}$ or $T^{3}$.

In this section, we give a justification for this phenomenon based on a heat kernel computation that recovers the result of Theorem 2.1 and justifies the presence of the factor $N / \# \Gamma$.

3.1. Generalities. We begin with some background on the spectral action for almost commutative spectral triples. In what follows, let $\mathscr{L}$ denote the Laplace transform, and let $S(0, \infty)=\{\phi \in S(\mathbb{R}) \mid \phi(x)=0, x \leq 0\}$.

Theorem 3.1. Let $(A, H, D)$ be a spectral triple of metric dimension $p$, and let $f: \mathbb{R} \rightarrow \mathbb{C}$ be even. If $|f(x)|=O\left(|x|^{-\alpha}\right)$ as $x \rightarrow \infty$ for some $\alpha>p$, then $f(D / \Lambda)=f(|D| / \Lambda)$ is trace-class for any $\Lambda>0$. If, in addition, $f(x)=$ $\mathscr{L}[\phi]\left(x^{2}\right)$ for some measurable $\phi: \mathbb{R}_{+} \rightarrow \mathbb{C}$, then

$$
\operatorname{Tr}(f(D / \Lambda))=\int_{0}^{\infty} \operatorname{Tr}\left(e^{-s D^{2} / \Lambda^{2}}\right) \phi(s) d s .
$$

Proof. Fix $\Lambda>0$. Let $\mu_{k}$ denote the $k$-th eigenvalue of $D^{2}$ in increasing order, counted with multiplicity; since $(A, H, D)$ has metric dimension $p,\left(\mu_{k}+1\right)^{-p / 2}=$ $O\left(k^{-1}\right)$ as $k \rightarrow \infty$, and hence for $k>\operatorname{dim} \operatorname{ker} D, \mu_{k}^{-1}=O\left(k^{-2 / p}\right)$ as $k \rightarrow \infty$. By our hypothesis on $f$, then, for $k>\operatorname{dim} \operatorname{ker} D$,

$$
\left|f\left(\mu_{k}^{1 / 2} / \Lambda\right)\right|=O\left(k^{-\alpha / p}\right), k \rightarrow \infty ;
$$

since $2 \alpha / p>1$, this implies that $\sum_{k=1}^{\infty} f\left(\mu_{k}^{1 / 2} / \Lambda\right)$ is absolutely convergent, as required.

Suppose, in addition, that $f(x)=\mathscr{L}[\phi]\left(x^{2}\right)$ for some measurable $\phi:[0, \infty) \rightarrow \mathbb{C}$. 
Then

$$
\begin{aligned}
\operatorname{Tr}(f(D / \Lambda)) & =\sum_{k=1}^{\infty} \mathscr{L}[\phi]\left(\mu_{k} / \Lambda^{2}\right) \\
& =\sum_{k=1}^{\infty} \int_{0}^{\infty} e^{-s \mu_{k} / \Lambda^{2}} \phi(s) d s \\
& =\int_{0}^{\infty}\left[\sum_{k=1}^{\infty} e^{-s \mu_{k} / \Lambda^{2}}\right] \phi(s) d s \\
& =\int_{0}^{\infty} \operatorname{Tr}\left(e^{-s D^{2} / \Lambda^{2}}\right) \phi(s) d s,
\end{aligned}
$$

as was claimed.

The above result raises the question of when a function $\phi: \mathbb{R}_{+} \rightarrow \mathbb{C}$ defines a function $f(x)=\mathscr{L}[\phi]\left(x^{2}\right)$ such that $f(D / \Lambda)$ is trace-class; a sufficient condition is given by the following lemma.

Lemma 3.2. If $\phi \in S(0, \infty)$, then $\mathscr{L}[\phi](s)=O\left(s^{-k}\right)$ as $s \rightarrow+\infty$ for all $k \in \mathbb{N}$.

Proof. Since $\phi \in S(0, \infty), \phi^{(k)}$ is a bounded function with $\phi^{(k)}(0)=0$ for all $k \in \mathbb{N}$, and hence $s^{n} \mathscr{L}[\phi](s)=\mathscr{L}\left[\phi^{(n)}\right](s)$ too is bounded, as required.

As a corollary of the above results, together with the asymptotic expansion of the heat kernel of a generalised Laplacian, we obtain the following basic result on the spectral action for almost commutative spectral triples:

Corollary 3.3 (cf. [26], Theorem 1). Let $\mathcal{V}$ be a self-adjoint Clifford module bundle on a compact oriented Riemannian manifold $M$, and let $D$ be a symmetric Dirac-type operator on $\mathcal{V}$. Let $f: \mathbb{R} \rightarrow \mathbb{C}$ be of the form $f(x)=\mathscr{L}[\phi]\left(x^{2}\right)$ for $\phi \in S(0, \infty)$. Then for $\Lambda>0, f(D / \Lambda)$ is trace-class with

$$
\operatorname{Tr}(f(D / \Lambda))=\int_{0}^{\infty}\left[\int_{M} \operatorname{tr}\left(K\left(s / \Lambda^{2}, x, x\right)\right) d \operatorname{Vol}(x)\right] \phi(s) d s,
$$

where $K(t, x, y)$ denotes the heat kernel of $D^{2}$, and $\operatorname{Tr} f(D / \Lambda)$ admits the asymptotic expansion

$$
\operatorname{Tr}(f(D / \Lambda)) \sim \sum_{k=-\operatorname{dim} M}^{\infty} \Lambda^{-k} \phi_{k} \int_{M} a_{k+\operatorname{dim} M}\left(x, D^{2}\right) d \operatorname{Vol}(x),
$$

as $\Lambda \rightarrow+\infty$, where $a_{n}\left(x, D^{2}\right)$ is the $n$-th Seeley-DeWitt coefficient of the generalised Laplacian $D^{2}$, and the constants $\phi_{n}$ are given by

$$
\phi_{n}=\int_{0}^{\infty} \phi(s) s^{n / 2} d s
$$


Proof. The first part of the claim follows immediately from the fact that

$$
\operatorname{Tr}\left(e^{-t D^{2}}\right)=\int_{M} \operatorname{tr}(K(t, x, x)) d \operatorname{Vol}(x), \quad t>0,
$$

while the second part of the claim follows immediately from the asymptotic expansion

$$
\operatorname{tr}(K(t, x, x)) \sim t^{-\operatorname{dim} M / 2} \sum_{k=0}^{\infty} t^{k / 2} a_{k}\left(x, D^{2}\right), \quad t \rightarrow 0,
$$

together with the assumption that $\phi$ has rapid decay, so that, in particular, the $\phi_{n}$ are all finite.

In fact, since $a_{n}\left(\cdot, D^{2}\right)=0$ for $n$ odd [15], Lemma 1.7.4, one has that the asymptotic form of $\operatorname{Tr}(f(D / \Lambda))$, as $\Lambda \rightarrow+\infty$, is given by

$$
\begin{cases}\sum_{n=0}^{\infty} \Lambda^{2(m-n)} \phi_{2(m-n)} \int_{M} a_{2 n}\left(x, D^{2}\right) d \operatorname{Vol}(x) & \text { if } \operatorname{dim} M=2 m, \\ \sum_{n=0}^{\infty} \Lambda^{2(m-n)+1} \phi_{2(m-n)+1} \int_{M} a_{2 n}\left(x, D^{2}\right) d \operatorname{Vol}(x) & \text { if } \operatorname{dim} M=2 m+1 .\end{cases}
$$

Note also that for $n>0$,

$$
\phi_{-n}=\int_{0}^{\infty} \phi(s) s^{-n / 2} d s=\frac{1}{\Gamma(n / 2)} \int_{0}^{\infty} f(u) u^{n-1} d u .
$$

The following result guarantees that the $\phi_{k}$ can be chosen at will:

Proposition 3.4. For any $\left(a_{n}\right) \in \mathbb{C}^{\mathbb{Z}}$ there exists some $\phi \in S(0, \infty)$ such that

$$
a_{n}=\int_{0}^{\infty} s^{n / 2} \phi(s) d s, \quad n \in \mathbb{Z} .
$$

In fact, this turns out to be a simple consequence of the following result by Durán and Estrada, solving the Hamburger moment problem for smooth functions of rapid decay:

Theorem 3.5 (Durán-Estrada, [14]). For any $\left(a_{n}\right) \in \mathbb{C}^{\mathbb{Z}}$ there exists some $\phi \in$ $\delta(0, \infty)$ such that

$$
a_{n}=\int_{0}^{\infty} s^{n} \phi(s) d s, \quad n \in \mathbb{Z} .
$$

Proof of Proposition 3.4. By Theorem 3.5, let $\psi \in S(0, \infty)$ be such that

$$
a_{n}=2 \int_{0}^{\infty} s^{n+1} \psi(s) d s, \quad n \in \mathbb{Z} .
$$

Then for $\phi(s)=\psi(\sqrt{s}) \in S(0, \infty)$,

$$
\int_{0}^{\infty} s^{n / 2} \phi(s) d s=2 \int_{0}^{\infty} t^{n+1} \psi(t) d t=a_{n}, \quad n \in \mathbb{Z},
$$

as required. 
3.2. Non-perturbative results. We now give a non-perturbative heat kernel-theoretic analysis of the phenomenon mentioned above.

Let $\tilde{M} \rightarrow M$ be a finite normal Riemannian covering with $\tilde{M}$ and $M$ compact, connected and oriented, and let $\Gamma$ be the deck group of the covering. Let $\widetilde{\mathcal{V}} \rightarrow \widetilde{M}$ be a $\Gamma$-equivariant self-adjoint Clifford module bundle, and let $\tilde{D}$ be a $\Gamma$-equivariant symmetric Dirac-type operator on $\widetilde{\mathcal{V}}$. We can therefore form the quotient self-adjoint Clifford modulebundle $\mathcal{V}:=\widetilde{\mathcal{V}} / \Gamma \rightarrow M=\widetilde{M} / \Gamma$, with $\widetilde{D}$ descending to a symmetric Dirac-type operator $D$ on $\mathcal{V}$; under the identification $L^{2}(M, \mathcal{V}) \cong L^{2}(\widetilde{M}, \widetilde{\mathcal{V}})^{\Gamma}$, we can identify $D$ with the restriction of $\widetilde{D}$ to $C^{\infty}(\tilde{M}, \widetilde{\mathcal{V}})^{\Gamma}$, where the unitary action $U: \Gamma \rightarrow U\left(L^{2}(\tilde{M}, \tilde{\mathcal{V}})\right)$ is given by $U(\gamma) \xi(\tilde{x}):=\xi\left(\tilde{x} \gamma^{-1}\right) \gamma$.

Our first goal is to prove the following result, relating the spectral action of $D$ to the spectral action of $\widetilde{D}$ in the high energy limit:

Theorem 3.6. Let $f: \mathbb{R} \rightarrow \mathbb{C}$ be of the form $f(x)=\mathscr{L}[\phi]\left(x^{2}\right)$ for $\phi \in S(0, \infty)$. Then for $\Lambda>0$,

$$
\operatorname{Tr}(f(D / \Lambda))=\frac{1}{\# \Gamma} \operatorname{Tr}(f(\tilde{D} / \Lambda))+O\left(\Lambda^{-\infty}\right), \quad \text { as } \Lambda \rightarrow+\infty .
$$

Remark 3.7. Theorem 3.6 continues to hold even when inner fluctuations of the metric are introduced, since for $A \in C^{\infty}(M$, $\operatorname{End}(\mathcal{V}))$ symmetric, $D+A$ on $\mathcal{V}$ lifts to $\widetilde{D}+\tilde{A}$ on $\tilde{\mathcal{V}}$, where $\tilde{A}$ is the lift of $A$ to $\tilde{\mathcal{V}}$.

To prove this result, we will need a couple of lemmas. First, we have the following well-known general fact:

Lemma 3.8. Let $G$ be a finite group acting unitarily on a Hilbert space $\mathcal{H}$, and let $A$ be a $G$-equivariant self-adjoint trace-class operator on $H$. Let $\mathscr{H}^{G}$ denote the subspace of $\mathscr{H}$ consisting of $G$-invariant vectors. Then the restriction $A \mid \mathscr{H}^{G}$ of $A$ to $\mathscr{H}^{G}$ is also trace-class and

$$
\operatorname{Tr}\left(A \mid \mathscr{H}^{G}\right)=\frac{1}{\# G} \sum_{g \in G} \operatorname{Tr}(g A) .
$$

Proof. This immediately follows from the observation that $\frac{1}{\# G} \sum_{g \in G} g$ is the orthogonal projection onto $\mathscr{H}^{G}$.

Now we can compute the heat kernel trace of $D$ using the heat kernel for $\widetilde{D}$ :

Lemma 3.9. For $t>0$,

$\operatorname{Tr}\left(e^{-t D^{2}}\right)=\frac{1}{\# \Gamma} \operatorname{Tr}\left(e^{-t \tilde{D}^{2}}\right)+\frac{1}{\# \Gamma} \sum_{\gamma \in \Gamma \backslash\{e\}} \int_{\tilde{M}} \operatorname{tr}\left(\rho(\gamma)\left(\tilde{x} \gamma^{-1}\right) \tilde{K}\left(t, \tilde{x} \gamma^{-1}, \tilde{x}\right)\right) d \operatorname{Vol}(\tilde{x})$,

where $\tilde{K}(t, \tilde{x}, \tilde{y})$ denotes the heat kernel of $\tilde{D}$ and $\rho$ denotes the right action of $\Gamma$ on the total space $\tilde{\mathcal{V}}$. 
Proof. Let $\gamma \in \Gamma$. Then

$$
\begin{aligned}
\left(U(\gamma) e^{-t \tilde{D}^{2}}\right) \xi(\tilde{x}) & =U(\gamma)\left(\int_{\tilde{M}} \tilde{K}(t, \tilde{x}, \tilde{y}) \xi(\tilde{y}) d \operatorname{Vol}(\tilde{y})\right) \\
& =\rho(\gamma)\left(\tilde{x} \gamma^{-1}\right)\left(\int_{\tilde{M}}\left(\tilde{x} \gamma^{-1}\right) \tilde{K}\left(t, \tilde{x} \gamma^{-1}, \tilde{y}\right) \xi(\tilde{y}) d \operatorname{Vol}(\tilde{y})\right) \\
& =\int_{\tilde{M}} \rho(\gamma)\left(\tilde{x} \gamma^{-1}\right) \tilde{K}\left(t, \tilde{x} \gamma^{-1}, \tilde{y}\right) \xi(\tilde{y}) d \operatorname{Vol}(\tilde{y})
\end{aligned}
$$

for any $\xi \in C^{\infty}(\tilde{M}, \tilde{\mathcal{V}})$, so that the operator $U(\gamma) e^{-t \widetilde{D}^{2}}$ has the integral kernel

$$
(t, \tilde{x}, \tilde{y}) \mapsto \rho(\gamma)\left(\tilde{x} \gamma^{-1}\right) \tilde{K}\left(t, \tilde{x} \gamma^{-1}, \tilde{y}\right) .
$$

Since $L^{2}(M, \mathcal{V}) \cong L^{2}(\tilde{M}, \tilde{\mathcal{V}})^{\Gamma}$, we can therefore apply Lemma 3.8 to obtain the desired result.

Finally, we can proceed with our proof.

Proof of Theorem 3.6. By Corollary 3.3 and Lemma 3.9, it suffices to show that for $\gamma \in G \backslash\{e\}$,

$$
\int_{0}^{\infty}\left[\int_{\tilde{M}} \operatorname{tr}\left(\rho(\gamma)\left(\tilde{x} \gamma^{-1}\right) \tilde{K}\left(s / \Lambda^{2}, \tilde{x} \gamma^{-1}, \tilde{x}\right)\right) d \operatorname{Vol}(\tilde{x})\right] \phi(s) d s=O\left(\Lambda^{-\infty}\right),
$$

as $\Lambda \rightarrow \infty$.

Now, since $\tilde{M}$ is compact and since the finite group $\Gamma$ acts freely and properly,

$$
\inf _{(\tilde{x}, \gamma) \in \tilde{M} \times \Gamma} d\left(\tilde{x} \gamma^{-1}, \tilde{x}\right)=\min _{(\tilde{x}, \gamma) \in \tilde{M} \times \Gamma} d\left(\tilde{x} \gamma^{-1}, \tilde{x}\right)>0 .
$$

Hence, by [16], Proposition 3.24, there exist constants $C>0, c>0$ such that

$$
\sup _{\tilde{x} \in \tilde{M}}\left\|\widetilde{K}\left(t, \tilde{x} \gamma^{-1}, \tilde{x}\right)\right\|_{2} \leq C e^{-c / t}, \quad t>0,
$$

for $\|\cdot\|_{2}$ the fibre-wise Hilbert-Schmidt norm, implying, in turn, that for every $n \in \mathbb{N}$ there exists a constant $C_{n}>0$ such that

$$
\sup _{\tilde{x} \in \tilde{M}}\left\|\tilde{K}\left(t, \tilde{x} \gamma^{-1}, \tilde{x}\right)\right\|_{2} \leq C_{n} t^{n}, \quad t>0 .
$$


Hence, for each $n \in \mathbb{N}$,

$$
\begin{aligned}
\mid \int_{0}^{\infty} & {\left[\int_{\tilde{M}} \operatorname{tr}\left(\rho(\gamma)\left(\tilde{x} \gamma^{-1}\right) \tilde{K}\left(s / \Lambda^{2}, \tilde{x} \gamma^{-1}, \tilde{x}\right)\right) d \operatorname{Vol}(\tilde{x})\right] \phi(s) d s \mid } \\
& \leq \int_{0}^{\infty} \operatorname{Vol}(M)\left(\sup _{\tilde{x} \in \tilde{M}}\|\rho(\gamma)(\tilde{x})\|_{2}\right)\left(\sup _{\tilde{x} \in \tilde{M}}\left\|\tilde{K}\left(s / \Lambda^{2}, \tilde{x} \gamma^{-1}, \tilde{x}\right)\right\|_{2}\right)|\phi(s)| d s \\
& \leq \operatorname{Vol}(M) \cdot\left(\sup _{\tilde{x} \in \tilde{M}}\|\rho(\gamma)(\tilde{x})\|_{2}\right) \cdot C_{n} \int_{0}^{\infty}\left(s / \Lambda^{2}\right)^{n}|\phi(s)| d s \\
& =\left(\operatorname{Vol}(M) \cdot\left(\sup _{\tilde{x} \in \tilde{M}}\|\rho(\gamma)(\tilde{x})\|_{2}\right) \cdot C_{n} \cdot \int_{0}^{\infty} s^{n}|\phi(s)| d s\right) \Lambda^{-2 n},
\end{aligned}
$$

yielding the desired result.

Now let $\alpha: \Gamma \rightarrow \mathrm{GL}_{N}(\mathbb{C})$ be a representation of $\Gamma$; by endowing $\mathbb{C}^{N}$ with a $\Gamma$-equivariant inner product, we take $\alpha: \Gamma \rightarrow U(N)$. Since $\tilde{M} \rightarrow M$ is a principal $\Gamma$-bundle, we form the associated Hermitian vector bundle $\mathcal{F}:=\tilde{M} \times{ }_{\alpha} \mathbb{C}^{N} \rightarrow M$; since $\Gamma$ is finite, we endow $\mathcal{F}$ with the trivial flat connection $d$. We can therefore form the self-adjoint Clifford module bundle $\mathcal{V} \otimes \mathcal{F} \rightarrow M$, which admits the symmetric Dirac-type operator $D_{\alpha}$ obtained from $D$ by twisting by $d$, that is,

$$
D_{\alpha}=D \otimes 1+c(1 \otimes d),
$$

where $c$ denotes the Clifford action on $\mathcal{V} \otimes \mathcal{F}$.

We now obtain the following generalisation of Theorem 3.6, which explains the factor of $N / \# \Gamma$ appearing in Theorem 2.1 above:

Theorem 3.10. Let $f: \mathbb{R} \rightarrow \mathbb{C}$ be of the form $f(x)=\mathscr{L}[\phi]\left(x^{2}\right)$ for $\phi \in S(0, \infty)$. Then for $\Lambda>0$,

$$
\operatorname{Tr}\left(f\left(D_{\alpha} / \Lambda\right)\right)=\frac{N}{\# \Gamma} \operatorname{Tr}(f(\widetilde{D} / \Lambda))+O\left(\Lambda^{-\infty}\right), \quad \text { as } \Lambda \rightarrow+\infty .
$$

Remark 3.11. This result is again compatible with inner fluctuations of the metric, insofar as if $A \in C^{\infty}(M$, $\operatorname{End}(\mathcal{V}))$ is symmetric, then $D_{\alpha}+A \otimes 1$ on $\mathcal{V} \otimes \mathscr{F}$ is induced from $\widetilde{D}+\tilde{A}$ on $\tilde{\mathcal{V}}$, where $\tilde{A}$ is $A$ viewed as a $\Gamma$-equivariant element of $C^{\infty}(\tilde{M}, \operatorname{End}(\widetilde{\mathcal{V}}))$.

Proof of Theorem 3.10. On the one hand, consider the trivial bundle $\widetilde{\mathcal{F}}:=\tilde{M} \times \mathbb{C}^{N}$ over $\tilde{M}$, together with the trivial flat connection $d$. Then for the action $(\tilde{x}, v) \gamma:=$ $\left(\tilde{x} \gamma, \alpha\left(\gamma^{-1}\right) v\right), \widetilde{F}$ is a $\Gamma$-equivariant Hermitian vector bundle, and $d$ is a $\Gamma$-equivariant Hermitian connection on $\widetilde{\mathcal{F}}$. Then, by taking the tensor product of $\Gamma$-actions, we can endow $\widetilde{\mathcal{V}} \otimes \widetilde{\mathcal{F}}$ with the structure of a $\Gamma$-equivariant self-adjoint Clifford module bundle, admitting the $\Gamma$-equivariant symmetric Dirac-type operator $\widetilde{D_{\alpha}}=\widetilde{D} \otimes 1+$ 
$c(1 \otimes d)$. As a vector bundle, however, we may simply identify $\tilde{\mathcal{V}} \otimes \widetilde{\mathcal{F}}$ with $\widetilde{\mathcal{F}} \oplus N$, in which case we may identify $\widetilde{D_{\alpha}}$ with $\widetilde{D} \otimes 1_{N}$.

On the other hand, by construction, the bundle $\mathscr{F}$ defined above is the quotient of $\widetilde{\mathcal{F}}$ by the action of $\Gamma$. Hence, under the action of $\Gamma$, the quotient of $\widetilde{\mathcal{V}} \otimes \widetilde{\mathcal{F}}$ is the self-adjoint Clifford module bundle $\mathcal{V} \otimes \mathscr{F}$, with $\widetilde{D_{\alpha}}$ descending to the operator $D \otimes 1+c(1 \otimes d)=D_{\alpha}$.

Finally, by Theorem 3.6 and our observations above,

$$
\begin{aligned}
\operatorname{Tr}\left(f\left(D_{\alpha} / \Lambda\right)\right) & =\frac{1}{\# \Gamma} \operatorname{Tr}\left(f\left(\widetilde{D_{\alpha}} / \Lambda\right)\right)+O\left(\Lambda^{-\infty}\right) \\
& =\frac{1}{\# \Gamma} \operatorname{Tr}\left(f(\widetilde{D} / \Lambda) \otimes 1_{N}\right)+O\left(\Lambda^{-\infty}\right) \\
& =\frac{N}{\# \Gamma} \operatorname{Tr}(f(\widetilde{D} / \Lambda))+O\left(\Lambda^{-\infty}\right), \quad \text { as } \Lambda \rightarrow+\infty,
\end{aligned}
$$

as was claimed.

One can apply these results to give a quick second proof of Theorem 2.1.

Second proof of Theorem 2.1. Recall that $\Gamma \subset \mathrm{SU}(2)$ is a finite group acting by isometries on $S^{3}$, identified with $\mathrm{SU}(2)$ endowed with the round metric, and that $\alpha: \Gamma \rightarrow U(N)$ is a representation. Since $S^{3}$ is parallelizable and $\Gamma$ acts by isometries, the spinor bundle $\mathbb{C}^{2} \rightarrow \delta_{S^{3}} \rightarrow S^{3}$ and the Dirac operator $\not D_{S^{3}}$ are trivially $\Gamma$-equivariant. Then, by construction, the Dirac-type operator $D_{\alpha}^{\Gamma}$ on $\delta_{S^{3}} \otimes \mathcal{V}_{\alpha}$ is precisely the induced operator $D_{\alpha}$ corresponding to $\widetilde{D}=\not D_{S^{3}}$, so that by Theorem 3.10,

$$
\operatorname{Tr}\left(f\left(D_{\alpha} / \Lambda\right)\right)=\frac{N}{\# \Gamma} \operatorname{Tr}\left(f\left(\not S_{S^{3}} / \Lambda\right)\right)+O\left(\Lambda^{-\infty}\right), \quad \text { as } \Lambda \rightarrow+\infty .
$$

However, by [6], §2.2, one has that

$$
\operatorname{Tr}\left(f\left(\not S_{S^{3}} / \Lambda\right)\right)=\Lambda^{3} \widehat{f^{(2)}}(0)-\frac{1}{4} \Lambda \hat{f}(0)+O\left(\Lambda^{-\infty}\right),
$$

where $\widehat{f^{(2)}}$ denotes the Fourier transform of $u^{2} f(u)$. Hence,

$$
\operatorname{Tr}\left(f\left(D_{\alpha} / \Lambda\right)\right)=\frac{N}{\# \Gamma}\left(\Lambda^{3} \widehat{f^{(2)}}(0)-\frac{1}{4} \Lambda \hat{f}(0)\right)+O\left(\Lambda^{-\infty}\right),
$$

as required.

3.3. Perturbative results. Let us now turn to the perturbative picture. In light of Corollary 3.3, it suffices to compare the Seeley-DeWitt coefficients of $\widetilde{D}^{2}$ with those of $D^{2}$ and $D_{\alpha}^{2}$. 
Proposition 3.12. Let $\widetilde{D}$ and $D$ be as above. Let $\pi: \tilde{M} \rightarrow M$ denote the quotient map. Then for all $n \in \mathbb{N}$,

$$
a_{n}\left(\pi(\tilde{x}), D^{2}\right)=a_{n}\left(\tilde{x}, \tilde{D}^{2}\right), \quad \tilde{x} \in \tilde{M},
$$

and hence

$$
\int_{M} a_{n}\left(x, D^{2}\right) d \operatorname{Vol}(x)=\frac{1}{\# \Gamma} \int_{\tilde{M}} a_{n}\left(\tilde{x}, \tilde{D}^{2}\right) d \operatorname{Vol}(\tilde{x}) .
$$

Proof. By [15], Lemma 4.8.1, there exist a unique connection $\nabla$ and endomorphism $E$ on $\mathcal{V}$ such that $D^{2}=\nabla^{*} \nabla-E$, and similarly a unique connection $\widetilde{\nabla}$ and endomorphism $\widetilde{E}$ on $\widetilde{\mathcal{V}}$ such that $\widetilde{D}^{2}=\widetilde{\nabla}^{*} \widetilde{\nabla}$. Since $\widetilde{D}^{2}$ is the lift of $D^{2}$ to $\widetilde{\mathcal{V}}$, it follows by uniqueness that $\widetilde{\nabla}$ and $\widetilde{E}$ are the lifts of $\nabla$ and $E$, respectively, to $\widetilde{\mathcal{V}}$ as well.

Now, since the finite group $\Gamma$ acts freely and properly on $\widetilde{M}$, let $\left\{\left(U_{\alpha}, \Psi_{\alpha}\right)\right\}$ be an atlas for $\tilde{M}$ such that for each $\alpha, \pi \mid U_{\alpha}: U_{\alpha} \rightarrow \pi\left(U_{\alpha}\right)$ is an isometry. Hence, the local data defining $a_{n}\left(\cdot, D^{2}\right)$ on $U_{\alpha}$ lifts to the local data defining $a_{n}\left(\cdot, \tilde{D}^{2}\right)$ on $\pi^{-1}\left(U_{\alpha}\right)$; since for $P=\nabla^{*} \nabla-E$ a generalised Laplacian on a Hermitian vector bundle $\varepsilon \rightarrow X$, each $a_{n}(\cdot, P)$ is given by a universal polynomial in the Riemannian curvature of $X$, the curvature of $\nabla, E$, and their respective covariant derivatives [15], $\S 4.8$, it therefore follows that $a_{n}\left(\cdot, \widetilde{D}^{2}\right)$ is indeed the lift to $\tilde{M}$ of $a_{n}\left(\cdot, D^{2}\right)$, as was claimed.

Proposition 3.13. Let $\widetilde{D}$ and $D_{\alpha}$ be as above. Then for all $n \in \mathbb{N}$,

$$
a_{n}\left(\pi(\tilde{x}), D_{\alpha}^{2}\right)=N a_{n}\left(\tilde{x}, \tilde{D}^{2}\right), \quad \tilde{x} \in \tilde{M}
$$

and hence

$$
\int_{M} a_{n}\left(x, D_{\alpha}^{2}\right) d \operatorname{Vol}(x)=\frac{N}{\# \Gamma} \int_{M} a_{n}\left(\tilde{x}, \tilde{D}^{2}\right) d \operatorname{Vol}(\tilde{x}) .
$$

Proof. On the one hand, by [15], Lemma 1.7.5, $a_{n}\left(\cdot, \widetilde{D}^{2} \otimes 1_{N}\right)=N a_{n}\left(\cdot, \widetilde{D}^{2}\right)$ for $\widetilde{D}^{2} \otimes 1_{N}$ on $\tilde{\mathcal{V}}^{\oplus N}$. On the other hand, $\widetilde{D}^{2} \otimes 1_{N}$ is the lift to $\tilde{\mathcal{V}}^{\oplus N}$ of $D_{\alpha}^{2}$ on $\mathcal{V} \otimes \mathcal{F}$, so that by Proposition $3.12, a_{n}\left(\cdot, \widetilde{D}^{2} \otimes 1_{N}\right)$ is the lift to $\tilde{M}$ of $a_{n}\left(\cdot, D_{\alpha}^{2}\right)$. Hence, $N a_{n}\left(\cdot, \widetilde{D}^{2}\right)$ is the lift to $\tilde{M}$ of $a_{n}\left(\cdot, D_{\alpha}^{2}\right)$, as required.

Let us now apply these results to the Dirac operator $\not_{S^{3}}$ on the round 3-sphere $S^{3}$, together with a finite subgroup $\Gamma$ of SU(2) acting freely and properly on $S^{3} \cong$ $\mathrm{SU}(2)$, and a representation $\alpha: \Gamma \rightarrow U(N)$. Since $\not D_{S^{3}}^{2}=\left(\nabla^{S}\right)^{*} \nabla^{S}+\frac{3}{2}$ by the Lichnerowicz formula, it follows from [15], Theorem 4.8.16, that

$$
\begin{aligned}
& \int_{S^{3}} a_{0}\left(x, \not D_{S^{3}}^{2}\right) d \operatorname{Vol}(x)=\int_{S^{3}}(4 \pi)^{-3 / 2} \operatorname{tr}(\mathrm{id}) d \operatorname{Vol}(x)=\frac{\sqrt{\pi}}{2} \\
& \int_{S^{3}} a_{2}\left(x, \not D_{S^{3}}^{2}\right) d \operatorname{Vol}(x)=\int_{S^{3}}(4 \pi)^{-3 / 2} \operatorname{tr}\left(\frac{6}{6} \mathrm{id}-\frac{3}{2} \mathrm{id}\right) d \operatorname{Vol}(x)=-\frac{\sqrt{\pi}}{4} .
\end{aligned}
$$


Since the operator $D_{\alpha}^{\Gamma}$ is precisely $D_{\alpha}$ as induced by $\widetilde{D}=\not D_{S^{3}}$, it therefore follows by Proposition 3.13 that

$$
\begin{aligned}
& \int_{S^{3} / \Gamma} a_{0}\left(y,\left(D_{\alpha}^{\Gamma}\right)^{2}\right) d \operatorname{Vol}(y)=\frac{N}{\# \Gamma} \int_{S^{3}} a_{0}\left(x, \not D_{S^{3}}^{2}\right) d \operatorname{Vol}(x)=\frac{N \sqrt{\pi}}{(\# \Gamma) 2}, \\
& \int_{S^{3} / \Gamma} a_{2}\left(y,\left(D_{\alpha}^{\Gamma}\right)^{2}\right) d \operatorname{Vol}(y)=\frac{N}{\# \Gamma} \int_{S^{3}} a_{2}\left(x, \not D_{S^{3}}^{2}\right) d \operatorname{Vol}(x)=-\frac{N \sqrt{\pi}}{(\# \Gamma) 4}
\end{aligned}
$$

Finally, one has

$$
\begin{aligned}
& \phi_{-3}=\frac{2}{\Gamma(3 / 2)} \int_{0}^{\infty} f(u) u^{2} d u=\frac{2}{\sqrt{\pi}} \int_{-\infty}^{\infty} f(u) u^{2} d u=\frac{2}{\sqrt{\pi}} \widehat{f^{(2)}}(0), \\
& \phi_{-1}=\frac{2}{\Gamma(1 / 2)} \int_{0}^{\infty} f(u) d u=\frac{1}{\sqrt{\pi}} \int_{-\infty}^{\infty} f(u) d u=\frac{1}{\sqrt{\pi}} \hat{f}(0),
\end{aligned}
$$

where $\widehat{f^{(2)}}$ is the Fourier transform of $f(u) u^{2}$. Hence,

$$
\begin{aligned}
\operatorname{Tr}\left(f\left(\not S_{S^{3}} / \Lambda\right)\right) & \sim \Lambda^{3} \phi_{-3} \int_{S^{3}} a_{0}\left(x, \not D_{S^{3}}^{2}\right) d \operatorname{Vol}(x) \\
& +\Lambda \phi_{-1} \int_{S^{3}} a_{2}\left(x, \not D_{S^{3}}^{2}\right) d \operatorname{Vol}(x)+O\left(\Lambda^{-1}\right) \\
& =\Lambda^{3} \widehat{f^{(2)}}(0)-\frac{1}{4} \Lambda \hat{f}(0)+O\left(\Lambda^{-1}\right)
\end{aligned}
$$

and

$$
\begin{aligned}
\operatorname{Tr}\left(f\left(D_{\alpha}^{\Gamma}\right)\right) \sim & \Lambda^{3} \phi_{-3} \int_{S^{3} / \Gamma} a_{0}\left(y,\left(D_{\alpha}^{\Gamma}\right)^{2}\right) d \operatorname{Vol}(y) \\
& +\Lambda \phi_{-1} \int_{S^{3} / \Gamma} a_{2}\left(y,\left(D_{\alpha}^{\Gamma}\right)^{2}\right) d \operatorname{Vol}(y)+O\left(\Lambda^{-1}\right) \\
= & \frac{N}{\# \Gamma}\left(\Lambda^{3} \widehat{f^{(2)}}(0)-\frac{1}{4} \Lambda \hat{f}(0)\right)+O\left(\Lambda^{-1}\right),
\end{aligned}
$$

which is indeed consistent with Theorem 2.1.

\section{The inflation potential and the power spectra}

It was shown in [20], [21] that for a 3-manifold $Y$ that is a spherical space form $S^{3} / \Gamma$ or a flat Bieberbach manifold (a quotient of the flat torus $T^{3}$ by a finite group action), the non-perturbative spectral action determines a slow-roll potential for a scalar field $\phi$ by setting

$$
\operatorname{Tr}\left(h\left(\left(D_{Y \times S^{1}}^{2}+\phi^{2}\right) / \Lambda^{2}\right)\right)-\operatorname{Tr}\left(h\left(D_{Y \times S^{1}}^{2} / \Lambda^{2}\right)\right)=V_{Y}(\phi),
$$


up to terms of order $O\left(\Lambda^{-\infty}\right)$, where, in the spherical space form case the potential is of the form

$$
V_{Y}(\phi)=\pi \Lambda^{4} \beta a^{3} \mathcal{V}_{Y}\left(\frac{\phi^{2}}{\Lambda^{2}}\right)+\frac{\pi}{2} \Lambda^{2} \beta a \mathcal{W}_{Y}\left(\frac{\phi^{2}}{\Lambda^{2}}\right),
$$

for $h$ the test function for the computation of the spectral action on the 4-manifold $Y \times S^{1}, a>0$ the radius of the sphere and $\beta>0$ the size of the circle compactification $S^{1}$. The functions $\mathcal{V}_{Y}$ and $\mathcal{W}_{Y}$ are of the form

$$
\mathcal{V}_{Y}(x)=\lambda_{Y} \mathcal{V}_{S^{3}}(x) \text { and } \mathcal{W}_{Y}(x)=\lambda_{Y} \mathcal{W}_{S^{3}}(x),
$$

where, for $Y=S^{3} / \Gamma$, the factor $\lambda_{Y}=(\# \Gamma)^{-1}$, and

$$
\mathcal{V}_{S^{3}}(x)=\int_{0}^{\infty} u(h(u+x)-h(u)) d u \quad \text { and } \quad \mathcal{W}_{S^{3}}(x)=\int_{0}^{x} h(u) d u .
$$

Thus, the potential satisfies

$$
V_{Y}(\phi)=\lambda_{Y} V_{S^{3}}(\phi)=\frac{V_{S^{3}}(\phi)}{\# \Gamma} .
$$

The slow-roll potential $V_{Y}(\phi)$ can be used as a model for cosmological inflation. As such, it determines the behavior of the power spectra $\mathcal{P}_{s, Y}(k)$ and $\mathcal{P}_{t, Y}(k)$ for the density fluctuations and the gravitational waves, respectively given in the form

$$
\mathcal{P}_{S}(k) \sim \frac{1}{M_{P l}^{6}} \frac{V^{3}}{\left(V^{\prime}\right)^{2}} \quad \text { and } \quad \mathcal{P}_{t}(k) \sim \frac{V}{M_{P l}^{4}},
$$

with $M_{P l}$ the Planck mass, see [32] and [21] for more details. Including second order terms, these can be written also as power laws as in [32],

$$
\begin{aligned}
& \mathcal{P}_{s}(k) \sim \mathcal{P}_{s}\left(k_{0}\right)\left(\frac{k}{k_{0}}\right)^{1-n_{s}+\frac{\alpha_{S}}{2} \log \left(k / k_{0}\right)}, \\
& \mathcal{P}_{t}(k) \sim \mathcal{P}_{t}\left(k_{0}\right)\left(\frac{k}{k_{0}}\right)^{n_{t}+\frac{\alpha_{t}}{2} \log \left(k / k_{0}\right)},
\end{aligned}
$$

where the exponents also depend on the slow roll potentials through certain slow-roll parameters. Since, as already observed in [20], [21], the slow-roll parameters are not sensitive to an overall multiplicative scaling factor in the potential, we focus here only on the amplitude only, which, as shown in [21], correspondingly changes by a multiplicative factor. Namely, in the case of a spherical space form with the spectral action computed for the untwisted Dirac operator, one has

$$
\begin{aligned}
& \mathcal{P}_{S, Y}(k) \sim \lambda_{Y} \mathcal{P}_{S}\left(k_{0}\right)\left(\frac{k}{k_{0}}\right)^{1-n_{s, S^{3}}+\frac{\alpha_{s, S^{3}}}{2} \log \left(k / k_{0}\right)}, \\
& \mathcal{P}_{t, Y}(k) \sim \lambda_{Y} \mathcal{P}_{t}\left(k_{0}\right)\left(\frac{k}{k_{0}}\right)^{n_{t, S^{3}}+\frac{\alpha_{t, S}}{2} \log \left(k / k_{0}\right)},
\end{aligned}
$$


where, as above, $\lambda_{Y}=1 / \# \Gamma$.

The amplitude and the exponents of the power law are parameters subject to constraints coming from cosmological observational data, as discussed in [18], [32], [33], so that, in principle, such data may be able to constrain the possible cosmic topologies in a model of gravity based on the spectral action. To this purpose, it is important to understand how much the amplitude and the slow-roll parameter are determined by the model. A discussion of the role of the parameters $\Lambda, a$, and $\beta$ is included in [21], while here we focus on how the coupling of gravity to matter affects these parameters.

By directly comparing the argument given in [20] proving (4.4) with the result of Theorem 2.1 above, we see that, in our case, we obtain then the following version of (4.4), modified by an overall multiplicative factor $N$, the total number of fermions in the model of gravity coupled to matter.

Proposition 4.1. For a spherical space form $Y=S^{3} / \Gamma$, consider the slow-roll potential $V_{Y, \alpha}(\phi)$ determined by the nonperturbative spectral action

$$
\operatorname{Tr}\left(h\left(\left(D_{\alpha, Y \times S^{1}}^{2}+\phi^{2}\right) / \Lambda^{2}\right)\right)-\operatorname{Tr}\left(h\left(D_{\alpha, Y \times S^{1}}^{2} / \Lambda^{2}\right)\right)=V_{Y, \alpha}(\phi),
$$

where $D_{\alpha, Y \times S^{1}}$ is the Dirac operator induces on the product geometry $Y \times S^{1}$ by the twisted Dirac operator $D_{\alpha}^{\Gamma}$ on $Y$. Then the associated power spectra as in (4.2), (4.3) satisfy (4.4), with $\lambda_{Y}=N / \# \Gamma$.

4.1. Inflation potential in the heat kernel approach. Let us now consider inflation potentials on space-times of the form $M \times S_{\beta}^{1}$ for $M$ compact oriented Riemannian and odd-dimensional, arising from general almost commutative triples over $M$.

Let $D$ is a symmetric Dirac-type operator on a self-adjoint Clifford module bundle $\mathcal{V} \rightarrow M$, with $M$ compact oriented Riemannian and odd-dimensional, and let $\not_{\beta}$ be the Dirac operator with simple spectrum $\frac{1}{\beta}\left(\mathbb{Z}+\frac{1}{2}\right)$ on the trivial spinor bundle $\mathbb{C} \rightarrow S_{S_{\beta}^{1}} \rightarrow S_{\beta}^{1}$. We may immediately generalise the construction of [6], §2.3, to obtain an odd symmetric Dirac-type operator $D_{M \times S_{\beta}^{1}}$ on the self-adjoint Clifford module bundle $\left(\mathcal{V} \otimes S_{S_{\beta}^{1}}\right)^{\oplus 2} \rightarrow M \times S_{\beta}^{1}$. Hence, we may define an inflation potential $V_{M}: C^{\infty}\left(M \times S_{\beta}^{1}\right) \rightarrow \mathbb{R}$ by

$$
V_{M}(\phi):=\operatorname{Tr}\left(h\left(\left(D_{M \times S_{\beta}^{1}}^{2}+\phi^{2}\right) / \Lambda^{2}\right)\right)-\operatorname{Tr}\left(h\left(D_{M \times S_{\beta}^{1}}^{2} / \Lambda^{2}\right)\right),
$$

where $h=\mathscr{L}[\psi]$ for $\psi \in S(0, \infty)$; note that $D_{M \times S_{\beta}^{1}}^{2}+\phi^{2}$ has heat trace

$$
\operatorname{Tr}\left(e^{-t\left(D_{M \times S_{\beta}^{1}}^{+}+\phi^{2}\right)}\right)=2 \operatorname{Tr}\left(e^{-t D_{\beta}^{2}}\right) \operatorname{Tr}\left(e^{-t D^{2}}\right) e^{-\phi^{2} t}
$$

for $\phi$ locally constant. 
Let $\Gamma \rightarrow \tilde{M} \rightarrow M, \tilde{\mathcal{V}} \rightarrow \tilde{M}, \mathcal{V} \rightarrow M, \widetilde{D}, D, \alpha, \mathcal{F} \rightarrow M$ and $D_{\alpha}$ be defined as in Subsection 3.2, with $M$ and $\tilde{M}$ odd-dimensional, generalising the discussion above of $\Gamma \rightarrow S^{3} \rightarrow Y$. On the one hand, we may form odd Dirac-type operators $\widetilde{D}_{\tilde{M} \times S_{\beta}^{1}}, D_{M \times S_{\beta}^{1}}$, and $D_{\alpha, M \times S_{\beta}^{1}}$ from $\widetilde{D}, D$ and $D_{\alpha}$, respectively, as above. On the other hand, if one trivially extends the action of $\Gamma$ on $\tilde{M}$ to $\tilde{M} \times S_{\beta}^{1}$ and the action on $\tilde{\mathcal{V}} \rightarrow \tilde{M}$ to $\left(\mathcal{V} \otimes S_{S_{\beta}^{1}}\right)^{\oplus 2} \rightarrow M \times S_{\beta}^{1}$, then $D_{\tilde{M} \times S_{\beta}^{1}}$ becomes a $\Gamma$-equivariant Dirac-type operator on $\left(\mathcal{V} \otimes S_{S_{\beta}^{1}}\right)^{\oplus 2}$, and the constructions of Section 3.2 applied to the $\Gamma$-equivariant Dirac-type operator $D_{\tilde{M} \times S_{\beta}^{1}}$ reproduce precisely the Dirac-type operators $D_{M \times S_{\beta}^{1}}$, and $D_{\alpha, M \times S_{\beta}^{1}}$.

Now let $V_{\tilde{M}}, V_{M}$ and $V_{M, \alpha}$ denote the inflation potentials corresponding to $\tilde{D}$, $D$ and $D_{\alpha}$, respectively, which we all view as nonlinear functionals on $C^{\infty}(\tilde{M} \times$ $\left.S_{\beta}^{1}, \mathbb{R}\right)^{\Gamma} \cong C^{\infty}\left(M \times S_{\beta}^{1}, \mathbb{R}\right)$. Then, since we also have that $D_{\tilde{M} \times S_{\beta}^{1}}^{2}+\phi^{2}$ is the lift of $D_{M \times S_{\beta}^{1}}^{2}+\phi^{2}$ and $\left(D_{\widetilde{M} \times S_{\beta}^{1}}^{2}+\phi^{2}\right) \otimes 1_{N}$ is the lift of $D_{M \times S_{\beta}^{1}, \alpha}^{2}+\phi^{2}$, Theorems 3.6 and 3.10, mutatis mutandis, therefore imply the following result:

Proposition 4.2. Let $\Gamma \rightarrow \tilde{M} \rightarrow M, \tilde{\mathcal{V}} \rightarrow X, \tilde{D}$ and $\alpha: \Gamma \rightarrow U(N)$ be as above, and define $\mathcal{V}, D, \mathcal{V}_{\alpha}$ and $D_{\alpha}$ as above. Let $V_{\tilde{M}}, V_{M}$ and $V_{M, \alpha}$ be defined as above. Then

$$
V_{M}(\phi)=\frac{1}{\# \Gamma} V_{\tilde{M}}(\phi)+O\left(\Lambda^{-\infty}\right), \quad \text { as } \Lambda \rightarrow+\infty
$$

and

$$
V_{M, \alpha}(\phi)=\frac{N}{\# \Gamma} V_{\tilde{M}}(\phi)+O\left(\Lambda^{-\infty}\right), \quad \text { as } \Lambda \rightarrow+\infty .
$$

This, therefore, explains the factor $\lambda_{Y}$ in eqs. (4.1) and (4.3) and Proposition 4.1.

Acknowledgment. This work is partially supported by NSF grants DMS-0901221, DMS-1007207, DMS-1201512, PHY-1205440. The second author thanks José Luis Cisneros-Molina for a useful conversation and for pointing out to us the results of [9], and the first author thanks Mathai Varghese for useful conversations.

\section{References}

[1] J. Boeijink and W. D. van Suijlekom, The noncommutative geometry of Yang-Mills fields. J. Geom. Phys. 61 (2011), 1124-1134. Zbl 1213.58020 MR 2782486

[2] M. Buck, M. Fairbairn, and M. Sakellariadou, Inflation in models with conformally coupled scalar fields: An application to the noncommutative spectral action. Phys. Rev. D 82 (2010), 043509. 
[3] B. Ćaćić, Moduli spaces of Dirac operators for finite spectral triples. In Quantum groups and noncommutative spaces, Aspects Math. E41, Vieweg Teubner, Wiesbaden 2011, 968. Zbl 1262.58017 MR 2798433

[4] B. Ćaćić, A reconstruction theorem for almost-commutative spectral triples. Lett. Math. Phys. 100 (2012), 181-202. Zbl 1253.58004 MR 2912480

[5] A. H. Chamseddine and A. Connes, The spectral action principle. Comm. Math. Phys. 186 (1997), 731-750. Zbl 0894.58007 MR 1463819

[6] A. H. Chamseddine and A. Connes, The uncanny precision of the spectral action. Comm. Math. Phys. 293 (2010), 867-897. Zbl 1192.58005 MR 2566165

[7] A. H. Chamseddine and A. Connes, Spectral action for Robertson-Walker metrics. $J$. High Energy Phys. 10 (2012), 101. MR 3033848

[8] A. H. Chamseddine, A. Connes, and M. Marcolli, Gravity and the standard model with neutrino mixing. Adv. Theor. Math. Phys. 11 (2007), 991-1089. Zbl 1140.81022 MR 2368941

[9] J. L. Cisneros-Molina, The $\eta$-invariant of twisted Dirac operators of $S^{3} / \Gamma$. Geom. Dedicata 84 (2001), 207-228. Zbl 0986.58008 MR 1825355

[10] A. Connes, Geometry from the spectral point of view. Lett. Math. Phys. 34 (1995), 203-238. Zbl 1042.46515 MR 1345552

[11] A. Connes, Gravity coupled with matter and the foundation of non-commutative geometry. Comm. Math. Phys. 182 (1996), 155-176. Zbl 0881.58009 MR 1441908

[12] A. Connes, On the spectral characterization of manifolds. J. Noncommut. Geom. 7 (2013), 1-82. Zbl 1287.58004 MR 3032810

[13] A. Connes and M. Marcolli, Noncommutative geometry, quantum fields and motives. Amer. Math. Soc. Colloq. Publ. 55, Amer. Math. Soc., Providence, RI, 2008. Zbl 1209.58007 MR 2371808

[14] A. L. Durán and R. Estrada, Strong moment problems for rapidly decreasing smooth functions. Proc. Amer. Math. Soc. 120 (1994), 529-534. Zbl 0795.44005 MR 1164143

[15] P. B. Gilkey, Invariance theory, the heat equation, and the Atiyah-Singer index theorem. 2nd ed., Studies in Advanced Mathematics, CRC Press, Boca Raton, FL 1995. Zbl 0856.58001 MR 1396308

[16] A. Kahle, Superconnections and index theory. Ph.D. thesis, University of Texas at Austin, 2008; J. Geom. Phys. 61 (2011), 1601-1624. Zbl 1225.58010 MR 2802496

[17] D. Kolodrubetz and M. Marcolli, Boundary conditions of the RGE flow in the noncommutative geometry approach to particle physics and cosmology. Phys. Lett. B 693 (2010), 166-174. MR 2720962

[18] J. E. Lidsey, A. R. Liddle, E. W. Kolb, E. J. Copeland, T. Barreiro, and M. Abney, Reconstructing the inflaton potential - an overview. Rev. Mod. Phys. 69 (1997), 373-410.

[19] M. Marcolli and E. Pierpaoli, Early universe models from noncommutative geometry. Adv. Theor. Math. Phys. 14 (2010), 1373-1432. Zbl 1243.83005 MR 2826185

[20] M. Marcolli, E. Pierpaoli, and K. Teh, The spectral action and cosmic topology. Comm. Math. Phys. 304 (2011), 125-174. Zbl 1216.83064 MR 2793932 
[21] M. Marcolli, E. Pierpaoli, and K. Teh, The coupling of topology and inflation in noncommutative cosmology. Comm. Math. Phys. 309 (2012), 341-369. Zbl 1242.83130 MR 2864797

[22] W. Nelson, J. Ochoa, and M. Sakellariadou, Constraining the noncommutative spectral action via astrophysical observations. Phys. Rev. Lett. 105 (2010) 101602.

[23] W. Nelson, J. Ochoa, and M. Sakellariadou, Gravitational waves in the spectral action of noncommutative geometry. Phys. Rev. D 82 (2010), 085021.

[24] W. Nelson and M. Sakellariadou, Cosmology and the noncommutative approach to the standard model. Phys. Rev. D 81 (2010), 085038.

[25] W. Nelson and M. Sakellariadou, Inflation mechanism in asymptotic noncommutative geometry. Phys. Lett. B 680 (2009), 263-266. MR 2574318

[26] R. Nest, E. Vogt, and W. Werner, Spectral action and the Connes-Chamsedinne model. In Noncommutative geometry and the standard model of elementary particle physics (Hesselberg, 1999), Lecture Notes in Phys. 596, Springer, Berlin 2002, 109-132. Zbl 1042.81106 MR 1998532

[27] A. Niarchou and A. Jaffe, Imprints of spherical nontrivial topologies on the cosmic microwave background. Phys. Rev. Lett. 99 (2007), 081302.

[28] P. Olczykowski and A. Sitarz, On spectral action over Bieberbach manifolds. Acta Phys. Polon. B 42 (2011), 1189-1198. MR 2806815

[29] A. Rennie and J. C. Varilly, Reconstruction of manifolds in noncommutative geometry. Preprint 2006. arXiv:math/0610418

[30] A. Riazuelo, J.-P. Uzan, R. Lehoucq, and J. Weeks, Simulating cosmic microwave background maps in multi-connected spaces. Phys. Rev. D 69 (2004), 103514.

[31] A. Riazuelo, J. Weeks, J.-P. Uzan, R. Lehoucq, J. P. Luminet, Cosmic microwave background anisotropies in multiconnected flat spaces. Phys. Rev. D 69 (2004), 103518.

[32] T. L. Smith, M. Kamionkowski, and A. Cooray, Direct detection of the inflationary gravitational-wave background. Phys. Rev. D 73 (2006), 023504.

[33] E. D. Stewart and D. H. Lyth, A more accurate analytic calculation of the spectrum of cosmological perturbations produced during inflation. Phys. Lett. B 302 (1993), 171-175.

[34] K. Teh, Nonperturbative spectral action of round coset spaces of SU(2). J. Noncommut. Geom. 7 (2013), 677-708. Zbl 1281.58020 MR 3108692

[35] T. van den Broek and W. D. van Suijlekom, Supersymmetric QCD and noncommutative geometry. Comm. Math. Phys. 303 (2011), 149-173. Zbl 1214.58004 MR 2775118

[36] W. D. van Suijlekom, Renormalization of the spectral action for the Yang-Mills system. J. High Energy Phys. 03 (2011), 146. MR 2821072

[37] W. D. van Suijlekom, Renormalization of the asymptotically expanded Yang-Mills spectral action. Comm. Math. Phys. 312 (2012), 883-912. Zbl 1247.58018 MR 2925137

Received December 15, 2011; revised June 26, 2012 
B. Ćaćić, Department of Mathematics, Mailstop 3368, Texas A\&M University, College Station, TX 77843-3368, U.S.A.

E-mail: branimir@math.tamu.edu

M. Marcolli, Mathematics Department, Mail Code 253-37, Caltech, 1200 E. California Blvd. Pasadena, CA 91125, U.S.A.

E-mail: matilde@caltech.edu

K. Teh, Mathematics Department, Mail Code 253-37, Caltech, 1200 E. California Blvd. Pasadena, CA 91125, U.S.A.

E-mail: teh@caltech.edu 